\title{
Pulsed Laser Interactions with Space Debris: Target Shape Effects
}

\author{
D. A. Liedahl, A. Rubenchik, S. B. Libby, S. Nikolaev \\ Lawrence Livermore National Laboratory \\ C. R. Phipps \\ Photonic Associates, LLC
}

\begin{abstract}
Among the approaches to the proposed mitigation and remediation of the space debris problem is the de-orbiting of objects in low Earth orbit through irradiation by ground-based high-intensity pulsed lasers. Laser ablation of a thin surface layer causes target recoil, resulting in the depletion of orbital angular momentum and accelerated atmospheric re-entry. However, both the magnitude and direction of the recoil are shape dependent, a feature of the laser-based remediation concept that has received little attention. Since the development of a predictive capability is desirable, we have investigated the dynamical response to ablation of objects comprising a variety of shapes. We derive and demonstrate a simple analytical technique for calculating the ablation-driven transfer of linear momentum, emphasizing cases for which the recoil is not exclusively parallel to the incident beam. For the purposes of comparison and contrast, we examine one case of momentum transfer in the low-intensity regime, where photon pressure is the dominant momentum transfer mechanism, showing that shape and orientation effects influence the target response in a similar, but not identical, manner. We address the related problem of target spin and, by way of a few simple examples, show how ablation can alter the spin state of a target, which often has a pronounced effect on the recoil dynamics.
\end{abstract}

Email addresses:

liedahl1@llnl.gov, rubenchik1@llnl.gov, libby1@llnl.gov, nikolaev2@llnl.gov

(D. A. Liedahl, A. Rubenchik, S. B. Libby, S. Nikolaev),

crphipps@photonicassociates.com (C. R. Phipps)

Preprint submitted to Advances in Space Research

August 22, 2018 
Keywords: laser ablation; laser orbit modification

\section{Introduction}

Over $90 \%$ of all objects in LEO represent space debris, including nonoperational spacecraft, rocket stages, mission-related debris, fragmentation debris, $\mathrm{NaK}$ coolant droplets, $\mathrm{Al}_{2} \mathrm{O}_{3}$ slag from solid rocket motor firings, etc. (Klinkrad, 2006). High-velocity impacts of operational payloads with debris objects as small as $1 \mathrm{~cm}$ in size and a few grams in mass can have potentially devastating effects: the on-orbit collisions produce more debris that increase the risk of subsequent orbital collisions, leading to a potential self-sustaining, runaway debris-creating process, known as the Kessler syndrome (Kessler and Cour-Palais, 1978, 1989). To counter the threat posed by the Kessler syndrome, several debris mitigation strategies have been proposed, including post-mission disposal of spacecraft, passivation of upper rocket stages, maneuvering into graveyard orbits for geostationary satellites, and improved collision avoidance.

Remediation (as distinct from mitigation) of the debris threat has also been proposed, and was given consideration under the auspices of NASA's Orion Project in the 1990s (Campbell, 1996a), in which a candidate remediation concept called for de-orbiting the debris using a ground-based laser (Phipps et al., 1996; Phipps, 1998). Predating the Orion Project, a somewhat more exotic technique involving a laser-mounted autonomous orbital vehicle had been suggested (Schall, 1991, 1998). In either case, the key concept is the creation of an ablation jet on the debris fragment, whereby orbital angular momentum can be removed, lowering the perigee and accelerating re-entry.

The Orion Project grouped the debris into five likely compositional classes: aluminum, steel, NaK metal, carbon phenolics, and insulation. Only the debris larger than approximately $10 \mathrm{~cm}$, about $5-10 \%$ of the total by number, can be tracked by radar and optical systems on a continuous basis. Nevertheless, there have been efforts to garner information related to smaller debris fragments; surveys in the radio and optical bands, augmented with modeling (Sdunnus et al., 2001), suggest that the population of objects in the $1-10 \mathrm{~cm}$ size range is $>150,000$, each member of which is too small to be monitored continuously, but any of which, with impact velocities of up to $16 \mathrm{~km} \mathrm{~s}^{-1}$, can debilitate or destroy spacecraft functionality. Since damage from collisions with objects smaller than about $1 \mathrm{~cm}$ can be mitigated against by shielding, 
it is the group of objects in the $1-10 \mathrm{~cm}$ size range that pose the greatest risk to space assets, but which, owing to their relatively small masses, can be favorably maneuvered to lower orbits with short re-entry times. Deflections of larger objects for the purpose of collision avoidance are also feasible (Phipps et al., 2012).

The current microscopic picture of laser debris coupling has its origin in studies of direct-drive physics relevant to inertial confinement fusion the picture developed (independently) by Kidder, Caruso, and Nemchimov (Kidder, 1971). For practical applications, the connection between the microphysics and the macrophysics is made by way of the mechanical coupling coefficient, denoted here by $C_{m}$. For a given element of surface of mass $m$ oriented such that its surface normal vector is labeled by $\hat{n}, C_{m}$ is defined so that the momentum change of a target responding to a laser energy deposition is given by

$$
m \Delta \vec{v}=-C_{m} E_{\text {inc }} \hat{n},
$$

where $E_{\text {inc }}$ is the total on-target laser energy. This expression contains the presumption that the net momentum vector of the ablation flow is parallel to the local surface normal.

Given Eq. 1, there is no reason to expect that momentum is transferred strictly along the laser propagation vector $\hat{k}$. However, that assumption is often used in estimating target recoil and subsequent orbital modifications, and constitutes one of the underpinnings of concept studies of debris clearing with lasers. The assumption that $\Delta \vec{v}$ and $\hat{k}$ are parallel simplifies calculations of the orbital dynamics and establishes a prescription for removing orbital angular momentum from the target: uprange irradiation, as close as possible to the horizon, after due consideration is given to geometrical dilution and atmospheric effects on the beam.

It is true that some objects do recoil in the beam direction; spheres, for example. Indeed, the concept of laser remediation, including the quantitative characterization of engagement strategies, is founded largely upon the assumption of spherical targets. While some fraction of the space debris population is likely to be spherical (see below), the actual shape distribution is uncertain, since evaluations of debris characteristics based on radar data do not include reliable shape assessments. Still, according to the NASA Technical Memorandum 108522 (Campbell, 1996b), periodicities of debris signatures have been noted, with periods ranging from about 0.1 second to tens of seconds. This suggests that at least some fraction of the debris is 
non-spherical or, at least, irregular. In any case, in light of Eq. 1, it is clear that a more general treatment of the problem is in order.

Given a differential element of area with a specific orientation characterized by its surface normal vector, the mechanical response following interaction with the laser is tied to (1) the orientation of the surface normal relative to the position vector with respect to Earth's center, which, in part, determines the angular momentum change to the orbit, and (2) the orientation of the surface normal with respect to the laser propagation vector, which determines the incident laser fluence and the impulse. We can anticipate a variety of possibilities: objects consisting essentially of two faces, such as a thin metallic plate, objects consisting of several faces, such as a rectangular solid, debris characterized by a continuously varying surface normal, such as a cylindrical or needle-like object, or irregularly-shaped objects that defy simple descriptions. In all these cases, the orbital solutions differ from the case of a spherical target.

Other laser-based schemes for space debris remediation or maneuvering must also accommodate shape effects, although the specifics can vary. For example, if, rather than surface ablation, photon pressure is the mechanism through which momentum is imparted to a target (Mason et al., 2011), then the recoil dynamics depend on the relative amounts of absorption, diffuse reflection, and specular reflection, the first transmitting momentum along the beam, and the other two adding off-beam components that are anti-parallel to the surface normal. Mason et al. (2011) argue that target spin will tend to average the specular reflection component to zero, and do not treat off-beam momentum transfer. However, we show below, explicitly for the ablation case, that the momentum impulse transmitted to a spinning object often retains a component transverse to the beam, and that the magnitude of this component is sensitive to the initial condition, i.e., the orientation of the target at the onset of laser illumination.

We have performed some preliminary work on the topic of shape effects (Liedahl et al., 2010), and expand upon it here. Our focus in this paper is on shape effects in the target rest frame. The consequences relevant to orbital modifications will be addressed in a followup paper. In $\S 2$, we develop the physical context of the problem and discuss some of the assumptions used in this paper. The basic approach for dealing with linear momentum transfer for arbitrary shapes is presented in $\S 3$. We present the results of a variety of calculations based on this methodology in $\S 4$. In $\S 5$, the related problem of target spin is addressed, again featuring a few examples. We conclude in $\S 6$, 
with a summary of our results, and suggestions for future refinements.

\section{A Few Preliminaries}

To better define the context of the problem, we derive some approximate scaling relations and discuss an approximation used throughout the paper.

\subsection{Estimate of the Required Velocity Change}

To obtain an estimate of the required velocity change, we consider the Hohmann transfer (Bate et al., 1971) from a circular orbit with radius $r$ to an elliptical orbit with perigee $r_{p}$, as shown in Fig. 1. From the the socalled vis viva equation, the orbital velocity of an object on an elliptical orbit around a mass $M$, taken here to be the mass of Earth, with semi-major axis $a$ is given by

$$
v^{2}=\frac{2 G M}{R}-\frac{G M}{a},
$$

when it is a distance $R$ from Earth's center. At the "transfer point," we thus have

$$
v_{1}^{2}=\frac{G M}{r} \quad v_{2}^{2}=\frac{2 G M}{r}-\frac{2 G M}{r+r_{p}},
$$

where the "1" and "2" denote "before (circle)" and "after (ellipse)," respectively. If $\Delta v=v_{2}-v_{1}$, then

$$
\frac{\Delta v}{v_{1}}=\left(\frac{2 r_{p}}{r+r_{p}}\right)^{1 / 2}-1
$$

Considering LEO only, and letting $\Delta r=r_{p}-r$, an adequate approximation is

$$
\Delta v \approx v_{1} \frac{\Delta r}{4 R_{E}} \approx 30 \frac{\Delta r}{100 \mathrm{~km}} \mathrm{~m} \mathrm{~s}^{-1}
$$

where $R_{E}$ is Earth's radius, and we set $v_{1}$ to $8000 \mathrm{~m} \mathrm{~s}^{-1}$. Therefore, LEO debris clearing requires velocity changes of up to a few hundred $\mathrm{m} \mathrm{s}^{-1}$, with the required $\Delta v$ directly (though approximately) proportional to the desired $\Delta r$. Note that it is also feasible to boost debris such that they achieve escape velocity. While perhaps not the best choice, since it merely displaces the problem, albeit into a much larger volume, it is in any case energetically expensive, since the required $\Delta v$ (in this case, positive) is approximately $\sqrt{2}-1$ times the orbital velocity, or about $3000 \mathrm{~m} \mathrm{~s}^{-1}$ for LEO. 


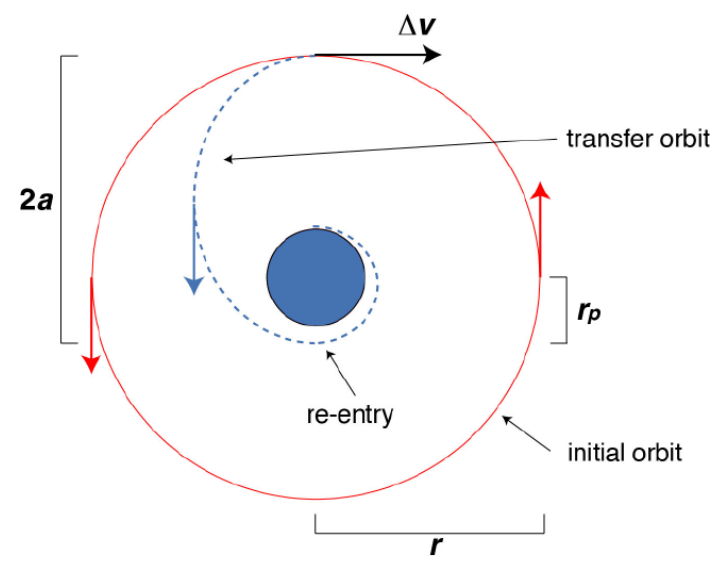

Figure 1: Geometry used to estimate the velocity change required to obtain a predetermined perigee via the initial stage of a Hohmann transfer (scales exaggerated for clarity). Starting from a counter-clockwise circular orbit (solid curve) with radius $r$, an impulse opposite the velocity vector, resulting in a velocity change $\Delta v$, is applied at the transfer point. The perturbed orbit (elliptical until capture; dashed curve) is characterized by its semi-major axis $a$ and perigee $r_{p}$, where $r_{p}$ is sufficiently low for re-entry to occur.

The estimate given above is just that, provided primarily to establish the regime in which we are working, and to serve as a useful benchmark for estimating laser energy requirements. Of course, one cannot simply choose to effect a classic Hohmann transfer from a ground station, since the momentum impulse given to an orbiting object will (typically) have components transverse to the velocity vector. We note the extreme case of a pure radial impulse, which does not remove any angular momentum, and, in fact, adds energy, but which nevertheless leads to a perigee reduction. Moreover, space debris orbits are not precisely circular. Considerations bearing on the efficient use of laser energy must make a distinction between engagement at descent and engagement at ascent. The various relations among orbital parameters, ground station coordinates, and target characteristics lead to a vast range of possibilities. Our interest here is to begin to characterize that range of possibilities, emphasizing the importance of target shape.

\subsection{Mass Loss}

The well-known rocket equation gives the velocity change of an object that has undergone continuous mass loss at a constant rate in a directed manner, 
with a mass ejection velocity $v_{a}$ in the rest frame of the object whose initial mass is $m_{o}$;

$$
\Delta v=v_{a} \ln \left(1-\Delta m / m_{o}\right)^{-1} \approx \frac{\Delta m}{m_{o}} v_{a},
$$

where the approximation is valid when the fractional mass loss is small. As is customary, in the model calculations presented here, we disregard the dynamical effects of material removal for all cases of linear momentum transfer.

Taken in its simplest form, active perigee reduction requires a change to the debris velocity $\Delta v$ as given in the previous subsection. Combining the approximation in Eq. 6 with the expression for $\Delta v$ from Eq. 5, we can estimate the fractional mass loss by

$$
\frac{\Delta m}{m} \approx \frac{v_{1}}{v_{a}} \frac{\Delta r}{4 R_{E}}
$$

The ejecta velocity $v_{a}$ depends on the material; it is higher for low-Z material than for heavy elements, and is about the thermal velocity of the surface plasma. To take an example, for Al $v_{a}$ is about $3-5 \mathrm{~km} \mathrm{~s}^{-1}$. Therefore, as an approximation, the fractional mass loss is

$$
\frac{\Delta m}{m} \approx 10^{-2} \frac{\Delta r}{100 \mathrm{~km}}
$$

Thus we can anticipate a total mass loss of order $1-10 \%$ of the initial mass for LEO de-orbiting campaigns. The fractional error introduced by using an approximate $\Delta v$, rather than the exact value $\Delta v_{\text {rocket }}$ is, from Eq. 6,

$$
\frac{\Delta v-\Delta v_{\text {rocket }}}{\Delta v_{\text {rocket }}}=-\frac{1}{2} \frac{\Delta m}{m_{o}}+O\left(\frac{\Delta m}{m_{o}}\right)^{2} .
$$

The ablated material expands, cools down, and forms nanoclusters (Zeldovich and Raizer, 2002), which are harmless. It is not clear, however, that ignoring shape deformation is "harmless" in terms of developing a predictive capability. Nevertheless, in the interest of keeping the calculations analytically manageable, we have not generally accounted for shape evolution during the course of an engagement.

We postpone our discussion of the treatment of mass loss in the context of angular momentum transfer until $§ 5.1$, after we develop the relevant analog of the force equation in the rotational case. 




Figure 2: The mechanical coupling coefficient for several materials (cellulose nitrate, polyoxymethylene, polystyrene) plotted as a function of $I \lambda \tau^{1 / 2}$, for intensity $I$, wavelength $\lambda$, and pulse duration $\tau$, illustrating the broad, slowly varying plateau in the region of maximum coupling, at intensities just above those that initiate plasma formation. The laser intensity corresponding to 10 on the $I \lambda \tau^{1 / 2}$ scale, near the peak in $C_{m}$, is $10^{9} \mathrm{~W}$ $\mathrm{cm}^{-2}$ for a ns pulse at laser wavelength of $1 \mu$, the value we assume here for numerical estimates. Figure from Sinko and Phipps (2009).

\subsection{Approximating the Coupling Coefficient}

Given that the emphasis of the paper is on shape effects, the magnitude of the coupling coefficient $C_{m}$ is not crucial. However, since the magnitude is known to depend on the incident intensity, and since we expect the intensity to vary across a non-planar surface, we need to justify an approximation used throughout the paper, namely, that $C_{m}$ can be treated as a constant across the illuminated surface of a given target.

A review of the experimental data for $C_{m}$ is presented by Phipps et al. (1988), and typical data are shown in Fig. 2 (Sinko and Phipps, 2009). As a function of laser intensity, $C_{m}$ is peaked not far beyond the vaporization threshold, where plasma starts to be generated. At low intensity levels, the 
surface temperature and evaporation rate are low, and the recoil momentum is relatively small - the coupling increases with intensity in this regime. At high intensity levels, a large fraction of the laser energy is used to create a plasma, which contributes little to the momentum change of the debris - the coupling decreases with intensity in this regime. Therefore, for some critical value of the laser intensity $I_{\text {crit }}, C_{m}$ is maximized. But, as is evident from Fig. 2, the maximum does not sharply define $I_{\text {crit }} ; C_{m}$ achieves a plateau over a broad range of laser intensity.

Since it is advantageous to work in the regime of maximum coupling, laser systems designed for ICF applications are well-suited to space debris clearing, as discussed in Rubenchik et al. (2012). In fact, data from different groups demonstrates that for a broad range of wavelength, pulse duration, and pulse energy, the coupling coefficient maximum is reached at an intensity

$$
I_{\text {crit }}=\frac{2.5}{\tau^{1 / 2}} \mathrm{GW} \mathrm{cm}^{-2},
$$

where $\tau$ is the pulse duration in nanoseconds (Phipps et al., 1988; Rubenchik et al., 2012). This points to high-powered short-pulse lasers. For intensities near $I_{\text {crit }}$ typical values of $C_{m}$ are 1-10 dyne $\mathrm{W}^{-1}$, and over the wide range of intensities near the peak, $C_{m}$ can be treated as being roughly constant. We adopt this approach below.

The experimental data correspond to planar targets only. It is fair to wonder if these data are applicable to calculations emphasizing non-planar targets. We note that for nanosecond-scale pulses the energy is absorbed in a thin surface layer, and the material is ejected normal to the surface. For ejecta velocities of about a few $\mathrm{km} \mathrm{s}^{-1}$, the ablation jet extends only a few microns during the pulse. Locally, the situation is equivalent to a flat surface.

\subsection{Estimate of the Required Laser Energy}

To relate $E_{\text {inc }}$ from Eq. 1 to the corresponding laser energy at the site of generation, the spot size is the crucial factor. In terms of the beam quality $Q$, the laser wavelength $\lambda$, the distance of the target from the laser site $d$, and the diameter of the beam director $D$, an expression for the spot size $a$ that accounts for beam diffraction is (Siegman, 1993)

$$
a=\frac{2 \lambda Q^{2} d}{\pi D} .
$$

As discussed by Mason et al. (2011), to approach the diffraction limit, a de-orbiting laser system would require the use of adaptive optics techniques 
with an artificial guide star, so as to mitigate against beam divergence caused by, for example, atmospheric turbulence (Beckers, 1993). Then, for a target of cross-sectional area $A$ as projected onto the beam direction, we have from Eq. 1

$$
E_{\mathrm{inc}}=\frac{E_{L} A}{\pi a^{2}}=\frac{m \Delta v}{C_{m}} .
$$

Assuming that the area-to-mass ratio is about $\left(\rho^{2} m\right)^{-1 / 3}$, we find from Eqs. 5,11 , and 12 that the required laser energy at the source can be expressed as

$$
E_{L}=\frac{\left(\rho^{2} m\right)^{1 / 3}}{\pi C_{m}} \frac{\lambda^{2} Q^{4}}{D^{2}} d^{2} v \frac{\Delta r}{R_{E}} .
$$

In order to provide a numerical estimate, we take a $100 \mathrm{~g}$ target, with $C_{m}=$ $10, \lambda=1 \mu, Q^{2}=2$, and scale to the remaining parameters, which gives

$$
E_{L} \sim 10^{3}\left(\frac{D}{6 \mathrm{~m}}\right)^{-2}\left(\frac{d}{500 \mathrm{~km}}\right)^{2}\left(\frac{\Delta r}{100 \mathrm{~km}}\right) \mathrm{kJ} .
$$

With a high rep-rate laser facility, such as those envisioned for future ICF power plants (Norimatsu et al., 2008; Dunne et al., 2011), operating at, say, $10 \mathrm{~Hz}$, with a $10 \mathrm{~kJ}$ per few-ns pulse, approximately $10^{4} \mathrm{~kJ}$ can be generated on a timescale comparable to a single-pass engagement (a few minutes), which compares favorably with Eq. 14, even if one chooses a less optimistic $C_{m}=1$.

\section{Transfer of Linear Momentum}

As discussed earlier, it is not valid to assume that the direction of the impulse following laser engagement is parallel to the beam, since that assumption is counter to the claim that ablation proceeds in a direction parallel to the surface normal. In this section we expand upon Eq. 1, and develop a methodology that will serve as a foundation for dealing with the general case of those irregularly-shaped objects that are likely to constitute a large fraction of the space debris population. While we deal strictly with idealized shapes, it is our aim to investigate some fundamental aspects of the problem that will provide some insight and form the basis of more advanced numerical studies.

Another case for which off-beam momentum transfer is important is that arising from interactions with targets that are larger than the beam itself. We do not treat this type of interaction in this paper, but rather assume 
throughout that the beam fully envelops the target. With beam radii of a few tens of cm (see Eq. 11), this is not particularly restrictive, since we are interested here in debris objects in the 1-10 cm range. Moreover, we ignore spatial variations in the beam intensity over the length scale of a debris surface, since, in the case of small debris, these variations are not large. Of course, one may choose to model the interaction with a higher degree of fidelity by including not just spatial variations of the beam (which implies a space-dependence to the mechanical coupling coefficient) but temporal profiles, as well. In the future, such detailed calculations may become warranted, but are unlikely to introduce new qualitative effects that would substantially alter the results presented here.

Proceeding, first we recast Eq. 1 as a force equation,

$$
m \frac{d \vec{v}}{d t}=-C_{m} \frac{d E_{\text {inc }}}{d t} \hat{n} .
$$

The laser intensity, as above, is denoted $I$ (energy per unit area per unit time). More rigorously, the quantity of interest is the flux. However, for a unidirectional beam, there is no important distinction between the scalar flux and the intensity. Nevertheless, we preserve the vector nature of the flux here, and, following convention, denote the intensity $\vec{I}=I \hat{k}$ for a unidirectional beam with propagation unit vector $\hat{k}$. Then, for a surface element of area $A$,

$$
\frac{d E_{\text {inc }}}{d t}=-A I \hat{k} \cdot \hat{n}
$$

The negative sign is required, since surface illumination can only occur for surface elements such that $\hat{k} \cdot \hat{n}<0$.

After substitution from Eq. 16, Eq. 15 becomes

$$
m \frac{d \vec{v}}{d t}=C_{m} I A \hat{k} \cdot \hat{n} \hat{n}
$$

which represents the response to irradiation of a single oriented surface. In general, to accommodate all irradiated surfaces, as well as cases where the surface normal varies continuously on a macroscopic level, we can define the dyadic form $\mathbf{G}$ according to

$$
\mathbf{G}=\sum_{\alpha} A_{\alpha} \hat{n}_{\alpha} \hat{n}_{\alpha} \rightarrow \int d A \hat{n} \hat{n}
$$


which we refer to as the area matrix. The momentum transfer equation or, force equation - can thus be written as

$$
m \frac{d \vec{v}}{d t}=C_{m} I \hat{k} \cdot \mathbf{G} .
$$

A matrix element $G_{i j}$ (note that $G_{i j}=G_{j i}$ ) describes the momentum impulse along the $j$-th coordinate direction resulting from the $i$-th component of the laser intensity vector. This could be the starting point for deriving a modified version of the rocket equation, but since we are working in the limit that the mass loss is small compared to the initial mass, we will accept Eq. 19 with $m$ constant, as implied. It should also be noted that $\mathbf{G}$ may be time-dependent; if any or all of the surface normals change with respect to a fixed coordinate system, then $\mathbf{G} \rightarrow \mathbf{G}(t)$.

\subsection{Approximating the Pulse Train}

We refer to a set of $N$ laser pulses with $N$ ablation events as an engagement. Roughly speaking, we assume that an engagement has a duration of tens of seconds, comprising some hundreds of individual pulses. Since we are considering nanosecond lasers with repetition rates of order 1-10 per second, it is a good approximation to represent the laser intensity as consisting of a set of delta functions, with pulses occurring at $t_{1}, t_{2}, \ldots, t_{N}$. However, it is simpler to treat the dynamical problem as though the intensity were constant in time throughout the engagement, in which case another approximation in the other direction, as it were - is called for. Letting $f$ denote the laser fluence per pulse at the target position (which we treat as a constant for a given engagement), the intensity can be written as

$$
\vec{I}=f \hat{k} \sum_{n=1}^{N} \delta\left(t-t_{n}\right),
$$

for a series of $N$ identical pulses - the pulse train. Substituting this expression for the pulse train into Eq. 19 gives

$$
\frac{d \vec{v}}{d t}=\frac{C_{m}}{m} f \hat{k} \cdot \sum_{n=1}^{N} \delta\left(t-t_{n}\right) \mathbf{G}(t),
$$

where, as mentioned above, we allow for the possibility that $\mathbf{G}$ is timedependent, as it may be for a rotating object. Starting from rest,

$$
\vec{v}(t)=\frac{C_{m}}{m} f \hat{k} \cdot \sum_{n=1}^{N} H\left(t-t_{n}\right) \mathbf{G}\left(t_{n}\right),
$$


where $H$ is the Heaviside step function.

In terms of the laser repetition rate $\nu$, we make the approximation

$$
\sum_{n} H\left(t-t_{n}\right) \rightarrow \nu \int d t,
$$

and define the average intensity $\bar{I}=f \nu$, so that

$$
\vec{v}(t)=\frac{C_{m}}{m} \bar{I} \hat{k} \cdot \int d t \mathbf{G}(t),
$$

or, working backwards,

$$
\frac{d \vec{v}}{d t}=\frac{C_{m}}{m} \bar{I} \hat{k} \cdot \mathbf{G}(t),
$$

where we recover Eq. 19 (now generalized). Problems involving both timeindependent and time-dependent variants of $\mathbf{G}$ are worked out below using Eq. 25.

For the remainder of the paper, we replace the symbol $\bar{I}$ by $I$, with the understanding that $I$ is the time-averaged magnitude of the laser intensity $(\bar{I}=I \nu \tau$ for a pulse duration $\tau$ of a few ns). To get a numerical estimate of $\bar{I}$, a typical fluence $f$ at the target is about $30 \mathrm{~J} \mathrm{~cm}^{-2}$, for a $10 \mathrm{~kJ}$ pulse and a spot radius of $10 \mathrm{~cm}$. If the laser pulse repetition rate $\nu$ is 10 per second, then the time-averaged intensity $f \nu \sim 300 \mathrm{~W} \mathrm{~cm}^{-2}$. Where needed, we use the value $100 \mathrm{~W} \mathrm{~cm}^{-2}$ below, keeping in mind that this average value is used in the force equations to reproduce the dynamics of a pulsed laser that has a much higher intensity during a pulse, so as to preserve the assumption of ablation near the peak range of the mechanical coupling coefficient.

One can invent hypothetical cases for which the assumption of a timeconstant beam will not apply. For example, suppose a debris object is rotating such that its period matches the laser pulse period, i.e., the object and the laser are "phase-locked." In that case, only one orientation of the object is sampled. Examples such as this can be worked out analytically. One finds that the net velocity change at the end of the laser engagement can be less than, greater than, or even equal to that obtained from using Eq. 25. Built into the time-constant-beam approach is the assumption that $\mathbf{G}$ is sampled continuously, rather than in discrete jumps. For this paper, which is intended as an exploration of some of the basic ideas, with a few worked-out examples to illustrate those ideas, we choose to avoid pathological cases, such as the one discussed here, and we restrict the calculations to those involving 
laser repetition rates that are substantially higher than the debris rotation rates. Further exploration of the wide range of possibilities may constitute worthwhile subjects of future efforts.

\section{Shape Effects on Translational Motion}

To demonstrate some of the consequences of Eq. 25, we provide a few examples using shapes that are, admittedly, highly idealized, in that we are unlikely to encounter many examples of such perfect objects in LEO. The examples are intended as a first foray into some of the anticipated phenomena associated with the laser/debris interaction that have yet to be addressed in detail.

\subsection{Cube}

The cube provides a useful example, as it demonstrates the utility of the area matrix. In the interest of emphasizing its relative ease of use, we first determine the momentum impulses on a cube in two orientations without invoking the area matrix technique. Consider a uniform cube of side $s$ and density $\rho$, so that $m=\rho s^{3}$. Orient the cube so that its edges are parallel or perpendicular to the axes of a Cartesian coordinate system, as in Fig. 3, so that the six surface normals coincide with the three Cartesian unit vectors and their opposites.

We evaluate the momentum impulse for two cases, one corresponding to the minimum projected area $s^{2}$, which occurs if the illumination is antiparallel to one of the six faces, and the other corresponding to the case of maximum projected area $s^{2} \sqrt{3}$, which occurs when the beam is along the main diagonal of the cube, and three faces are simultaneously illuminated. Intuition would suggest that various orientations of the cube should result in a complex variety of momentum impulses. Additionally, the incident laser energy depends on the orientation; one might expect the maximum magnitude of the impulse to correspond to the maximum projected area. As we now show, this is not the case.

In either case, fix the laser such that $\hat{k}=-\hat{z}$. The case of single-surface ablation requires no rotation of the cube, whereas the down-the-diagonal case does. Note that in the context of this paper one can use the equivalent procedure of "moving" the laser to access any case of interest, i.e., one can freely select $\hat{k}$. However, in the larger context of calculating the momentum impulse of a debris target that is on orbit, $\hat{k}$ is dictated by the laser-to-target 




Figure 3: Homogeneous cube of mass $m$, mass density $\rho$, and with edge length $s$, with three of the six surface normals illustrated. In one example (see text), the laser is incident from above $(\hat{k}=-\hat{z})$, so that only the face corresponding to $\hat{n}_{z}$ is illuminated. In a second example, the cube is rotated so that the main diagonal is parallel to $\hat{k}$, i.e., $\hat{z} \rightarrow \hat{z}^{\prime}$, and three faces are illuminated.

position vector - there is no leeway. Thus it is conceptually simpler to rotate the object in a fixed coordinate system. We use the latter, albeit somewhat more algebraically involved, approach here.

Referring to Fig. 3, only the top surface is illuminated in the minimumprojected-area case, with the surface normal $(0,0,1)$. Equation 17 gives us

$$
\frac{d \vec{v}}{d t}=-\frac{C_{m} I s^{2}}{m} \hat{z}=\frac{C_{m} I s^{2}}{m} \hat{k}
$$

For the second case, we construct a rotation matrix as the product of two coordinate rotation matrices, the first obtained by rotation about the $z$-axis by the angle $\phi=-\pi / 4$ and the second by rotating about the $x^{\prime}$ axis (which is subsequently discarded) by the angle $\theta=-\arccos (1 / \sqrt{3})$. These are the first two of the three Euler angles using the " $x$-convention" (Goldstein, 1980). Explicitly, the net rotation proceeds as follows:

$$
R=R_{\theta} R_{\phi}=\frac{1}{\sqrt{3}}\left(\begin{array}{rrr}
\sqrt{3} & 0 & 0 \\
0 & 1 & -\sqrt{2} \\
0 & \sqrt{2} & 1
\end{array}\right) \times \frac{1}{\sqrt{2}}\left(\begin{array}{rrr}
1 & -1 & 0 \\
1 & 1 & 0 \\
0 & 0 & \sqrt{2}
\end{array}\right)
$$


which leaves

$$
R=\frac{1}{\sqrt{6}}\left(\begin{array}{rrr}
\sqrt{3} & -\sqrt{3} & 0 \\
1 & 1 & -2 \\
\sqrt{2} & \sqrt{2} & \sqrt{2}
\end{array}\right)
$$

which moves the main diagonal such that it lies along the $z$-axis.

Operating with $R$ on the three surface normals gives

$$
\hat{n}_{x}^{\prime}=\frac{(\sqrt{3}, 1, \sqrt{2})}{\sqrt{6}} \quad \hat{n}_{y}^{\prime}=\frac{(-\sqrt{3}, 1, \sqrt{2})}{\sqrt{6}} \quad \hat{n}_{z}^{\prime}=\frac{(0,-2, \sqrt{2})}{\sqrt{6}}
$$

These constitute the set of illuminated surface normals of our rotated cube in the fixed Cartesian coordinate system.

Again, using Eq. 17, the acceleration components are

$$
\frac{d v_{i}}{d t}=\frac{C_{m} I s^{2}}{m} \sum_{\alpha}\left(\hat{k} \cdot \hat{n}_{\alpha}^{\prime}\right)\left(\hat{x}_{i} \cdot \hat{n}_{\alpha}^{\prime}\right)
$$

which for this case gives the results

$$
\begin{gathered}
\frac{d v_{x}}{d t}=-\frac{C_{m} I s^{2}}{\sqrt{3} m} \times \frac{1}{\sqrt{6}}(\sqrt{3}-\sqrt{3})=0 \\
\frac{d v_{y}}{d t}=-\frac{C_{m} I s^{2}}{\sqrt{3} m} \times \frac{1}{\sqrt{6}}(1+1-2)=0 \\
\frac{d v_{z}}{d t}=-\frac{C_{m} I s^{2}}{\sqrt{3} m} \times \frac{1}{\sqrt{6}}(\sqrt{2}+\sqrt{2}+\sqrt{2})=-\frac{C_{m} I s^{2}}{m}
\end{gathered}
$$

The final result for the acceleration vector when illumination is down the main diagonal is thus

$$
\frac{d \vec{v}}{d t}=\frac{C_{m} I s^{2}}{m}(0,0,-1)=\frac{C_{m} I s^{2}}{m} \hat{k}=\frac{1}{\left(\rho^{2} m\right)^{1 / 3}} C_{m} I \hat{k}
$$

which is identical to the case of single-surface normal-incidence illumination (Eq. 26). Moreover, as can be shown, one obtains the same result for any orientation of the cube. This result is at odds with a scalar version of the momentum equation, since, if that were correct, the recoil would be proportional to the area of the cube projected onto the beam direction, which determines the amount of intercepted laser energy. Mathematically, this can be understood as follows. Since, in the coordinate system of Fig. 3, the area 
matrix before rotation is $\mathbf{G}=s^{2} \mathbf{I}$, where $\mathbf{I}=\operatorname{diag}(1,1,1)$, any rotation of the object will leave $\mathbf{G}$ unchanged. For any object, if $\mathbf{G} \propto \mathbf{I}$, then

$$
R \cdot \mathbf{G} \cdot R^{-1} \propto R \cdot \mathbf{I} \cdot R^{-1}=\mathbf{I},
$$

and, from Eq. 19, $d \vec{v} / d t \propto \hat{k} \cdot \mathbf{G} \| \hat{k}$ regardless of orientation. Thus in general, if $\mathbf{G}$ takes the form of a multiple of the unit tensor in one orientation, it takes that form in all orientations, and the recoil is along the beam.

By contrast to the somewhat laborious calculation by which we arrived at Eq. 34, one can use Eq. 19 to immediately obtain the correct result for an arbitrarily oriented cube. Qualitatively, the result expresses the compensating trade-offs in the competition between the projected areas of the three faces and the transmission of momentum along the three coordinate axes (the projections of the surface normals onto the coordinate axes), including the effects of cancellation or reinforcement. The relative convenience of the area matrix is evident.

\subsection{Sphere}

Since a large proportion of earlier work related to de-orbiting small debris objects has assumed a spherical shape, we take as our next example a solid, homogeneous sphere of radius $R$ and mass density $\rho$. In fact, $\mathrm{NaK}$ coolant spheres are believed to constitute a non-negligible fraction of the space debris population (Wiedemann, et al., 2005). In any case, this example serves as a straightforward application of the area matrix approach.

Let the center of the sphere be coincident with the origin of a Cartesian coordinate system, with the normal vector given in terms of standard spherical coordinates according to

$$
\hat{n}=(\sin \theta \cos \phi, \sin \theta \sin \phi, \cos \theta),
$$

and the differential element of solid angle $d \Omega=\sin \theta d \theta d \phi$. Using the integral form of Eq. 18, the area matrix is

$$
\mathbf{G}=R^{2} \int d \Omega\left(\begin{array}{ccc}
\sin ^{2} \theta \cos ^{2} \phi & \sin ^{2} \theta \sin \phi \cos \phi & \sin \theta \cos \theta \cos \phi \\
\sin ^{2} \theta \sin \phi \cos \phi & \sin ^{2} \theta \sin ^{2} \phi & \sin \theta \cos \theta \sin \phi \\
\sin \theta \cos \theta \cos \phi & \sin \theta \cos \theta \sin \phi & \cos ^{2} \theta
\end{array}\right)
$$

With no loss of generality, we can assume that $\hat{k}=\hat{z}$, so that the limits of integration, which range only over the illuminated portion of the sphere, are 
$\theta \in[\pi / 2, \pi]$ and $\phi \in[0,2 \pi]$. By inspection, all off-diagonal elements vanish. The diagonal elements are all equal to $2 \pi R^{2} / 3$, so that

$$
\mathbf{G}=\frac{2}{3} \pi R^{2} \operatorname{diag}(1,1,1)
$$

simply the unit tensor times a geometrical factor. Therefore, from Eq. 25, the force equation is

$$
m \frac{d \vec{v}}{d t}=\frac{2}{3} \pi R^{2} C_{m} I \hat{k} .
$$

Not surprisingly, the impulse is along $\hat{k}$. Given the form of this equation, it is fair to say that, in this context, the effective area of a sphere is $(2 / 3) \pi R^{2}$, i.e., smaller than the geometrical cross-section.

Since we have assumed that the sphere is a homogeneous solid, we eliminate $R$ in favor of $m$ and $\rho$, according to $R=(3 m / 4 \pi \rho)^{1 / 3}$, which, after substitution into Eq. 39, leaves

$$
\frac{d \vec{v}}{d t}=\left(\frac{\pi}{6 \rho^{2} m}\right)^{1 / 3} C_{m} I \hat{k} .
$$

which is analogous to the far right-hand side of Eq. 34 for a cube. This shows that a "scale-up" to a larger mass, for a given density, goes as $m^{-1 / 3}$, and that $\left(\rho^{2} m\right)^{-1 / 3}$ can be seen as an approximation to the effective areato-mass ratio, with a correction term of $(\pi / 6)^{1 / 3}$ for a sphere. Therefore, in considering the relative plausibility of substantially modifying the orbits of, let us say, a $1 \mathrm{~g}$ object and a $10^{6} \mathrm{~g}$ object, one should work with a scale factor of $10^{-2}$, rather than $10^{-6}$ when evaluating the relative velocity change. Moreover, the internal mass distributions of large objects are not homogeneous; the density $\rho$ appearing in Eq. 40 is not the material density but rather the average density of the entire body, thereby adding to the plausibility of maneuvering large objects. One caveat here is that, for this kind of scaling, the laser beam must overfill the target, since the projected area, assumed to be fully illuminated, enters into the calculation.

To touch base with the earlier approximate $\Delta v$ requirement set by Eq. 5 , we take the two examples thus far derived and consider two homogeneous aluminum targets $\left[C_{m}=2\right.$ dynes $\mathrm{W}^{-1}$; a conservative representative value taken from P. Combis, et al. (personal communication)], each with a mass of $100 \mathrm{~g}$. With an average intensity of $100 \mathrm{~W} \mathrm{~cm}^{-2}$ (§3.1), we find from Eqs. 34 and 40 ,

$$
\Delta v=0.18 \Delta t \mathrm{~m} \mathrm{~s}^{-1} \text { (sphere) } \Delta v=0.23 \Delta t \mathrm{~m} \mathrm{~s}^{-1} \text { (cube) }
$$


if $\Delta t$ is given in seconds. The difference is a consequence of the fact that the effective area-to-mass ratio of a cube is larger than that of a sphere of equal mass by the factor $(6 / \pi)^{1 / 3}$. Thus a $100 \mathrm{~km}$ perigee reduction requires an engagement duration of 100-200 seconds for these two objects, attainable on a single pass, assuming a laser system as outlined in $§ 2.4$, and as discussed in more detail in Rubenchik et al. (2012). Reacquisition and engagement over multiple orbits may be required in many cases, where larger perigee changes are desired.

The previous numerical estimate is provided only as a means to reinforce feasibility studies of the basic methodology. Obviously, the efficacy of perigee reduction depends on the direction of $\Delta v$, not just its magnitude. Suffice to say that for these two shapes $\Delta \vec{v}$ is along the beam, and it has been shown for this case that the optimum engagement angle is approximately $30^{\circ}$ uprange of laser zenith (Rubenchik et al., 2012), in which case a substantial radial impulse may be imparted to the orbiting debris fragment. In the following examples, the impulse does not lie exclusively along the beam and the issue of engagement optimization is an open question.

\subsection{Plate}

The simplest example of an object that will exhibit an off-beam response to laser ablation is a flat plate oriented such that the surface normal has a component orthogonal to the beam direction. The situation is as illustrated in Fig. 4 , with the angle $\phi \in[0, \pi]$ describing the orientation relative to edgeon. We assume that the plate has, in effect, only two dimensions, i.e., we neglect the edges. The laser is incident from the left, such that $\hat{k}=\hat{x}$. The particular shape of the plate used in this example - circular, square, random - is not important, as long as it is thin in one dimension and perfectly flat. Only the surface area matters.

The surface normal is given by

$$
\hat{n}=\left(\begin{array}{c}
-\sin \phi \\
\cos \phi
\end{array}\right),
$$

where the $z$-component is omitted, since it plays no role in this example. The area matrix (again, no $z$-components) is, therefore,

$$
\mathbf{G}=A\left(\begin{array}{cc}
\sin ^{2} \phi & -\sin \phi \cos \phi \\
-\sin \phi \cos \phi & \sin ^{2} \phi
\end{array}\right),
$$




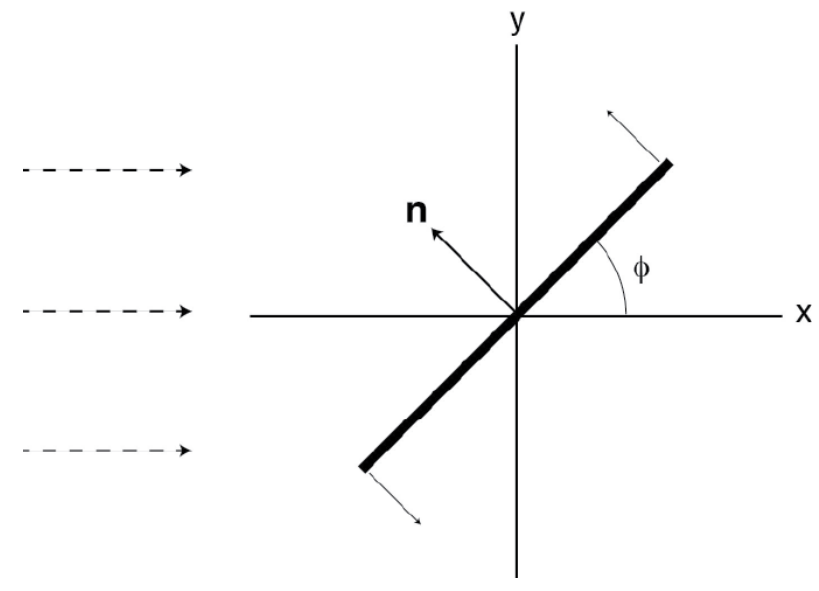

Figure 4: Geometric setup showing a thin plate projected onto the $x y$-plane. Laser light (dashed arrows) is incident from the left. As described in $\S 4.3$, we first assume that the plate is not spinning. In $\S 4.5$, we let the plate spin counter-clockwise at a constant angular velocity $(\omega=\omega \hat{z})$.

from which

$$
\left(\begin{array}{c}
\dot{v}_{x} \\
\dot{v}_{y}
\end{array}\right)=\frac{C_{m} I A}{m}\left(\begin{array}{c}
\sin ^{2} \phi \\
-\sin \phi \cos \phi
\end{array}\right),
$$

or

$$
\frac{d \vec{v}}{d t}=-\frac{C_{m} I A}{m} \sin \phi \hat{n}
$$

Not surprisingly, the trajectories are straight lines with the displacement varying with $t^{2}$. The maximum $x$-component of velocity corresponds to the face-on orientation, the maximum $y$-component of velocity corresponds to $\phi=\pi / 4$ or $3 \pi / 4$, owing to the competing effects of projected area and alignment of the normal along the $\hat{y}$ direction, and the maximum total velocity corresponds to the face-on configuration.

\subsection{Plate Responding to Photon Pressure}

At low surface intensities, below the threshold for melting, the kinematic response of an illuminated object is dominated by photon pressure. A scheme for space debris maneuvering using photon pressure is presented by Mason et al. (2011). Although somewhat off-topic, the situation bears similarities to the pure ablation case, so we provide a brief treatment here, restricting the discussion to this single example of a flat plate. The problem is similar 




Figure 5: Momentum vector relationships for laser illumination of a flat plate in the photon pressure regime. It assumed that the beam overfills the target. Reflection transfers momentum to a direction opposite the surface normal $\mathbf{n}$, while absorption transfers momentum along the incident beam direction $\mathbf{k}$. The magnitude of the momentum transferred along $\mathbf{k}$ decreases with increasing albedo $\alpha$. The quantity $\beta$ is the fraction of reflected light in the specular component. We assume that the diffuse radiation is hemispherically distributed, with a net vector along the normal. The figure shows that, typically, the net momentum acquired by the target is neither along the beam nor precisely opposite the normal.

to that of pure ablation, although slightly more complex at the macroscopic level, since the relative amounts of absorption, specular reflection, and diffuse reflection all play a role; absorption and reflection transmit momentum in two different directions, unless the beam happens to be aligned with the surface normal.

The situation is illustrated in Figure 5. The fraction of incident light that is reflected is determined by the albedo $\alpha$. The fraction of reflected light that goes into the specular component is denoted by $\beta$, so that the diffuse reflection fraction is given by $\alpha(1-\beta)$. The direction of the specular reflection component is denoted $\hat{k}^{\prime}$ and is given in terms of the incident beam direction 
and the surface normal by

$$
\hat{k}^{\prime}=\hat{k}-2(\hat{k} \cdot \hat{n}) \hat{n}
$$

Momentum conservation gives

$$
m \frac{d \vec{v}}{d t}=\frac{I A}{c}|\hat{k} \cdot \hat{n}|\left[\hat{k}-\alpha \beta \hat{k}^{\prime}-\frac{1}{2} \alpha(1-\beta) \hat{n}\right],
$$

The factor of $1 / 2$ represents our assumption that the diffuse reflection component originating at a given point on the surface is distributed uniformly into a hemisphere. Referred to the coordinate system in Fig. 4, the $x$ and $y$ force equations are

$$
\begin{gathered}
\dot{v}_{x}=\frac{I A}{m c} \sin \phi\left[1-\alpha \beta+\frac{1}{2} \alpha(1-\beta) \sin \phi+2 \alpha \beta \sin ^{2} \phi\right] \\
\dot{v}_{y}=-\frac{I A}{m c} \alpha \sin \phi \cos \phi\left[\frac{1}{2}(1-\beta)+2 \beta \sin \phi\right]
\end{gathered}
$$

Excepting the case where the beam is aligned with the normal, it is only in the case of pure absorption $(\alpha=0)$ that the momentum transfer is strictly along the beam $\left(\dot{v}_{y}=0\right)$, in which case the force equation becomes

$$
\dot{v}_{x}=\frac{I A}{m c} \sin \phi \quad \text { (pure absorption). }
$$

At the other extreme - pure specular reflection $(\alpha=1, \beta=1)$ — we find

$$
\frac{d \vec{v}}{d t}=2 \frac{I A}{m c} \sin ^{2} \phi\left(\begin{array}{c}
\sin \phi \\
-\cos \phi
\end{array}\right) \quad \text { (pure specular reflection). }
$$

From Eq. 42, we see that the expression for pure specular reflection can also be written as an acceleration along the normal;

$$
\frac{d \vec{v}}{d t}=-2 \frac{I A}{m c} \sin ^{2} \phi \hat{n} \quad \text { (pure specular reflection), }
$$

where we recover the magnitude of the recoil $(2 I A / m c)$ and the "effective mechanical coupling coefficient" $\left(2 / c=6.7 \times 10^{-4}\right.$ dyne $\left.\mathrm{W}^{-1}\right)$ in the case of normal incidence. One can compare this expression to the analogous case of pure ablation (Eq. 45). The dependence on the incident angle differs in the two cases. In the photon pressure case, an additional power of $\sin \phi$ appears 
owing simply to Snell's Law, whereas ablation does not obey the rules of optics in this case. Thus the efficiency of the photon pressure mechanism drops rapidly as grazing incidence $(\phi \rightarrow 0)$ is approached.

We note one further effect. Since a fraction of the laser energy is absorbed, the target will re-radiate this energy. In the rest frame of a continuously irradiated thin plate, with illuminated surface 1 and the "dark" surface 2, reradiation (subscript $\mathrm{RR}$ ) constitutes an additional contributor to the normal component of the momentum transfer;

$$
\left(\dot{v}_{n}\right)_{\mathrm{RR}}=-\frac{A}{m c}\left(I_{1}-I_{2}\right) .
$$

Since $I_{1} \geq I_{2}$, this momentum component is also opposite to the surface normal.

In general, determining $I_{1}$ and $I_{2}$, even for this simple geometry, is a complicated problem in non-linear heat diffusion, and we pursue it no further here. We can evaluate the two limiting cases: (1) $I_{2}=I_{1}$ (no added momentum), and (2) $I_{2}=0$, in which case

$$
\left(\frac{d \vec{v}}{d t}\right)_{\mathrm{RR}}=\frac{I_{1} A}{m c}\left(\begin{array}{c}
\sin \phi \\
-\cos \phi
\end{array}\right) .
$$

The re-radiation intensity is highly material-dependent. In lieu of an exact solution, the result for the maximum re-radiation impulse (Eq. 54) can be added provisionally to Eqs. 48 and 49, where it becomes evident that it may be of a magnitude comparable to the other contributors, and favorably modifies the photon pressure scheme for engaging space debris. Reliable quantitative evaluations await detailed numerical calculations.

\subsection{Spinning Plate}

Returning now to pure ablation pressure, suppose the plate described in $\S 4.3$ is spinning around an axis perpendicular to a surface normal, such that the spin axis intersects the center of mass, as shown in Fig. 4, and that the spin frequency $\omega$ is unaffected by ablation. Suppressing the $z$-component, which plays no role here, the surface normal is

$$
\hat{n}=\left(\begin{array}{c}
-\sin (\omega t+\phi) \\
\cos (\omega t+\phi)
\end{array}\right)
$$


The time-dependent area matrix (again, no $z$-components) is, therefore,

$$
\mathbf{G}(t)=A\left(\begin{array}{cc}
\sin ^{2}(\omega t+\phi) & -\sin (\omega t+\phi) \cos (\omega t+\phi) \\
-\sin (\omega t+\phi) \cos (\omega t+\phi) & \sin ^{2}(\omega t+\phi)
\end{array}\right)
$$

from which

$$
\left(\begin{array}{c}
\dot{v}_{x} \\
\dot{v}_{y}
\end{array}\right)=\frac{C_{m} I A}{m}\left(\begin{array}{c}
\sin ^{2}(\omega t+\phi) \\
-\sin (\omega t+\phi) \cos (\omega t+\phi)
\end{array}\right)
$$

With the initial condition $\left(v_{x}, v_{y}\right)=(0,0)$, the $x$ and $y$ velocity components are

$$
\begin{gathered}
v_{x}=\frac{C_{m} I A}{2 m \omega}[\omega t-\sin \omega t \cos (\omega t+2 \phi)] \\
v_{y}=-\frac{C_{m} I A}{2 m \omega} \sin \omega t \sin (\omega t+2 \phi)
\end{gathered}
$$

By spin-averaging the previous expression for $v_{y}$, we find that

$$
\left\langle v_{y}\right\rangle=-\frac{C_{m} I A}{4 m \omega} \cos 2 \phi,
$$

which can be thought of as an average drift velocity - the presence of spin does not generally obviate the need for considering off-beam velocity components. Although the $y$-component of the impulse is indeed oscillatory, with an average magnitude of zero, the target drifts. However, the resultant trajectory is sensitive to the value of $\phi$ at the onset of ablation. When $\phi=(2 n-1) \pi / 4(n=1,2,3, \ldots)$, the drift term vanishes. In all other cases, either a positive or negative drift velocity orthogonal to the beam is present.

The trajectories are approximately parabolic $\left(x \propto y^{2}\right)$, as can be inferred from Eqs. 58 and 60, i.e., if ablation continues indefinitely, then the trajectory approaches a parabola, with linear eccentricity $q$ (defined so that $y^{2}=4 q x$ ) given by

$$
q=\frac{C_{m} I A \cos ^{2} 2 \phi}{16 m \omega^{2}}
$$

which shows that the parabola "narrows" as $\omega^{-2}$ - for sufficiently large $\omega$, a straight-line approximation becomes progressively more tenable. On shorter time scales, the trajectories are somewhat complex, and depend sensitively on the phase angle, as shown in Fig. 6. Note also the lack of trajectory symmetry; comparing $\phi=45^{\circ}$ and $\phi=135^{\circ}$, the former travels farther, since it gets a "head start" at early times, rotating into a more complete alignment with the laser field. 


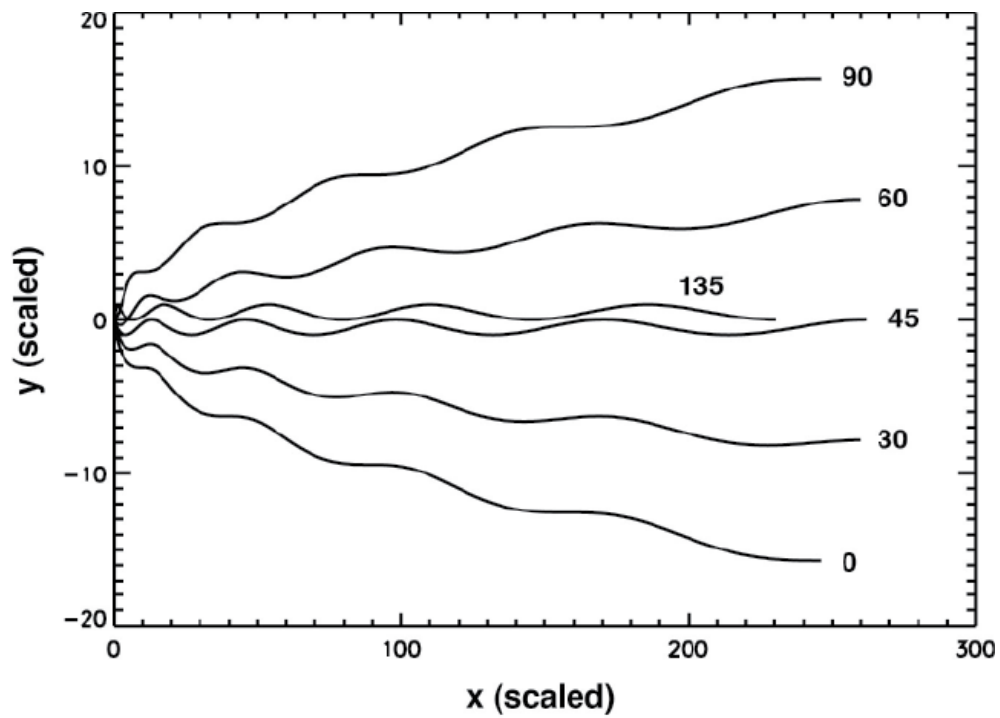

Figure 6: Trajectories of the center of mass of a spinning plate for six different initial phase angles (as indicated), covering 2.5 rotation cycles. Axes are scaled (see text).

\subsection{Cylinder}

We now examine a solid, homogeneous cylinder of mass density $\rho$, with radius $r$ and height $h$, supposing that the cylinder has circular end caps of the same material as the cylinder walls. A particular mode of cylinder rotation will be considered, but first, to calculate $\mathbf{G}$, let the cylindrical axis be aligned with the $z$-axis. After determining $\mathbf{G}$ in this orientation, the more general form is found through a rotational transformation below. Using the standard cylindrical coordinates $(\varrho, \phi, z)$, we have $\varrho=(\cos \phi, \sin \phi, 0)$. The area matrix can be constructed as the sum of two components, with only one (or neither) end cap illuminated.

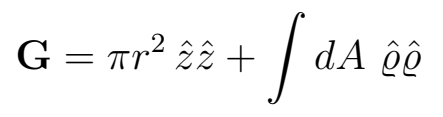

Since

$$
\hat{\varrho} \hat{\varrho}=\left(\begin{array}{ccc}
\cos ^{2} \phi & \sin \phi \cos \phi & 0 \\
\sin \phi \cos \phi & \sin ^{2} \phi & 0 \\
0 & 0 & 0
\end{array}\right),
$$





Figure 7: Relative orientation of a solid cylinder with respect to a laser beam propagating along the $y$-axis, described by the angle $\psi$, with the cylindrical axis restricted to the $y z$ plane. At most, one end cap is illuminated. With the orientation and dimensions shown, the recoil will have a positive $y$-component and a negative $z$-component. As the $h / r$ ratio is reduced the $z$-component switches from negative to positive, passing through zero when $h=2 r(a=1)$.

and we integrate $z$ over $[-h / 2, h / 2]$ and $\phi$ over $[\pi, 2 \pi]$, we find

$$
\mathbf{G}=\frac{\pi}{2}\left(\begin{array}{ccc}
r h & 0 & 0 \\
0 & r h & 0 \\
0 & 0 & 2 r^{2}
\end{array}\right) \equiv \pi r^{2}\left(\begin{array}{ccc}
a & 0 & 0 \\
0 & a & 0 \\
0 & 0 & 1
\end{array}\right)
$$

where $a \equiv h / 2 r$. Note that the special case $h=2 r(a=1)$ leaves $\mathbf{G}$ as a multiple of the unit tensor, so that the recoil is strictly along the beam in this special case, regardless of orientation, i.e., regardless of the $\hat{k}$-direction, the recoil will always be along $\hat{k}$. In fact, the two ranges $a<1$ and $a>1$ result in qualitatively different responses, which motivates the introduction of $a$.

Let the laser propagate along the $y$-axis $(\hat{k}=\hat{y})$. Rather than allowing freedom in $\hat{k}$, we introduce a degree of freedom to allow for a range of cylinder orientations, viz., we allow rotation about the $x$-axis by an angle $\psi \in[0, \pi]$ (see Fig. 7), which may be time dependent. In practice, it may be easier to calculate the recoil by fixing the object and "moving" the laser. However, when coupled with the orbital calculations, it is more natural to fix the laser in an Earth-centered coordinate system and let the object rotate in this 
system. Although the orbital mechanics aspects of the problem are being deferred to a later paper, we choose that approach here.

To rotate the corresponding area matrix in this fixed coordinate system, such that the cylindrical axis always lies in the $y z$-plane, we use the transformation $\mathbf{G}_{\text {rot }}=R \mathbf{G} R^{-1}$, where the rotation matrix $R$ is

$$
R=\left(\begin{array}{ccc}
1 & 0 & 0 \\
0 & \cos \psi & \sin \psi \\
0 & -\sin \psi & \cos \psi
\end{array}\right)
$$

The rotated area matrix is thus given by

$$
\mathbf{G}_{\mathrm{rot}}=\pi r^{2}\left(\begin{array}{ccc}
a & 0 & 0 \\
0 & a \cos ^{2} \psi+\sin ^{2} \psi & -(a-1) \sin \psi \cos \psi \\
0 & -(a-1) \sin \psi \cos \psi & a \sin ^{2} \psi+\cos ^{2} \psi
\end{array}\right)
$$

Using our definition of $a$, we eliminate $r=(m / 2 \pi \rho a)^{1 / 3}$, so as to recast the following development in terms of $\left(\rho^{2} m\right)^{-1 / 3}$, as above, and the equation of motion is

$$
\frac{d \vec{v}}{d t}=\left(\frac{\pi}{4 \rho^{2} m a^{2}}\right)^{1 / 3} C_{m} I\left(\begin{array}{c}
0 \\
a \cos ^{2} \psi+\sin ^{2} \psi \\
-(a-1) \sin \psi \cos \psi
\end{array}\right)
$$

The $y$-component of force, hence velocity, is always positive, whereas the $z$-component may be positive, negative, or zero. There are two special cases: $\psi=0$ and $\psi=\pi / 2$. If $\psi=0$, the endcaps are not illuminated, and there is no $z$-component to the impulse. For the $y$-component, the impulse can, by analogy to the spherical case discussed earlier, be associated with an effective cross-section, which in this case is $(\pi / 2) r h$, again smaller than the projected area $2 r h$. When $\psi=\pi / 2$, only the endcap is illuminated, and again there is no $z$-component to the impulse vector. As for any flat surface whose normal is anti-parallel to $\hat{k}$, the effective area is equal to its actual geometrical area of $\pi r^{2}$. Other than for the "sphere-like" $a=1$ case, if $\psi$ is not an integer multiple of $\pi / 2$, the force equation shows a $z$-component, and the motion is not parallel to $\hat{k}$.

Proceeding, let us consider a case in which the cylinder rotates about the $x$ axis at a constant angular velocity $\omega$ (clockwise in Fig. 7), so that $\psi=\omega t+\phi$, where $\phi$ sets the orientation at the moment the laser first illuminates the 
cylinder; for reference, if $\phi=0$ at $t=0$, the initial orientation is such that the cylindrical axis is aligned with the $z$-axis.

With the initial conditions $v_{y}=v_{z}=0$, the velocity solutions of Eq. 67 are

$$
\begin{gathered}
v_{y}=2 \frac{a+1}{a^{2 / 3}} \kappa \omega\left[\omega t+\frac{a-1}{a+1} \sin \omega t \cos (\omega t+2 \phi)\right] \\
v_{z}=-2 \frac{a-1}{a^{2 / 3}} \kappa \omega \sin \omega t \sin (\omega t+2 \phi)
\end{gathered}
$$

where $\kappa$, with the dimension of length, is defined by

$$
\kappa \equiv \frac{C_{m} I}{4 \omega^{2}}\left(\frac{\pi}{4 \rho^{2} m}\right)^{1 / 3}
$$

In a manner similar to the case of a rotating plate, the rotating cylinder has a drift velocity in a direction orthogonal to the beam: from Eq. 69, it is easy to show that the spin-averaged drift velocity is

$$
\left\langle v_{z}\right\rangle=-\frac{a-1}{a^{2 / 3}} \kappa \omega \cos 2 \phi
$$

which vanishes only when $\phi=(2 n-1) \pi / 4(n=1,2,3, \ldots)$, identical to the case of a plate. The magnitude of the drift velocity is at its maximum whenever $\phi$ is an integer multiple of $\pi / 2$. The leading term for $v_{y}$ (along the beam) is independent of $\omega$, while $\left\langle v_{z}\right\rangle \propto \omega^{-1}$.

Neglecting the oscillatory second term of Eq. 68, one finds that the minimum $y$-component of the velocity occurs for the case $a=2$, i.e., for a cylinder with the approximate relative dimensions found for a (U.S.A. standard) soda can. This detail is related neither to the $a$-dependence of the average projected area, which decreases monotonically as $a^{-2 / 3}$ for a fixed volume, nor to the minimum absolute area for a fixed volume $(a=1)$. The maximum attainable velocity corresponds to either limit $a \rightarrow 0$ or $a \rightarrow \infty$, which becomes unphysical, i.e., an infinite disk of vanishing thickness in the former case, or an infinitely long rod of vanishing radius in the latter.

With the initial conditions $y=z=0$ for the cylinder's center of mass, the coordinate solutions of Eq. 67 are

$$
\begin{gathered}
y(t)=\frac{a+1}{a^{2 / 3}} \kappa\left[(\omega t)^{2}-\frac{a-1}{a+1}(\omega t \sin 2 \phi-\sin \omega t \sin (\omega t+2 \phi))\right] \\
z(t)=-\frac{a-1}{a^{2 / 3}} \kappa[\omega t \cos 2 \phi-\sin \omega t \cos (\omega t+2 \phi)]
\end{gathered}
$$





Figure 8: Trajectories of non-spinning, solid cylinders for four values of $a=h / 2 r$ (as indicated in upper right of each panel), for equal-mass cylinders of height $h$ and radius $r$, for ten different orientations. Axes are scaled, as described in text.

As expected, spin has no effect on the center-of-mass motion when $a=$ 1 , since then all terms containing $\omega$ drop out. In the limit $\omega t \gg 1$ the trajectories become parabolic.

The behavior of a non-spinning cylinder ( $\psi$ fixed) is found by taking the limit $\omega \rightarrow 0$ (also, $\psi=\phi$ in this case),

$$
\begin{gathered}
v_{y}(t ; \omega=0)=\left(\frac{\pi}{4 \rho^{2} m}\right)^{1 / 3}\left[\frac{a+1+(a-1) \cos 2 \psi}{2 a^{2 / 3}}\right] C_{m} I t \\
v_{z}(t ; \omega=0)=-\left(\frac{\pi}{4 \rho^{2} m}\right)^{1 / 3} \frac{(a-1) \sin 2 \psi}{2 a^{2 / 3}} C_{m} I t
\end{gathered}
$$

With the same initial conditions $y=z=0$, the coordinate solutions are

$$
\begin{gathered}
y(t ; \omega=0)=\left(\frac{\pi}{32 \rho^{2} m}\right)^{1 / 3} \frac{a+1+(a-1) \cos 2 \psi}{2 a^{2 / 3}} C_{m} I t^{2} \\
z(t ; \omega=0)=-\left(\frac{\pi}{32 \rho^{2} m}\right)^{1 / 3} \frac{(a-1) \sin 2 \psi}{2 a^{2 / 3}} C_{m} I t^{2}
\end{gathered}
$$


(Of course, the non-spinning solution may also be found directly by solving Eq. 67 with $\psi$ fixed.) The trajectories are straight lines for all $t$, but have a marked dependence on both $a$ and $\psi$, as shown in Fig. 8..

Recalling that the $a=1$ case leads to "sphere-like" motion of the cylinder, it should be made clear that this is true only in the sense that the recoil is along $\hat{k}$ in either case. Comparing the recoil velocities, we see from Eqs. 40 and 74 that the velocity of the $a=1$ cylinder is $(3 / 2)^{1 / 3} \approx 1.14$ times that of a sphere, assuming identical masses, mass densities, and coupling coefficients.

\section{Transfer of Spin Angular Momentum}

In the previous section, we added spin to a thin plate and to a cylinder, assuming that the spin is independent of the laser/target interaction. In this section, we examine the interaction directly, with the aim of characterizing the extent to which laser ablation induces or otherwise affects spin. While it is unlikely that all potential targets are spinning - for example, spin may be damped by Earth's magnetic field (Praly et al., 2012) — some are. Moreover, in some cases, the laser interaction may itself induce spin.

Since ablation causes a local force per unit area on an object, a torque about the center of mass may result. Here we assume that the force points exactly opposite the local surface normal, as in earlier sections. If the areal summation of these local torques is non-zero, the spin angular momentum of the object may be altered. We refer to this as the "static torque" (symbolized by $N_{s}$ ), since it applies whether or not the target is spinning at the onset of the laser interaction. The symmetry aspects of the target figure prominently here, since certain symmetries ensure that for every torque there is another that is equal but opposite, and spin cannot be induced; for example, a circular disk, or a uniform rod. However, with the exception of NaK spheres, there is no reason to expect that space debris possess any kind of symmetry.

The static torque is given by

$$
\vec{N}_{s}=C_{m} I \int d A(\hat{k} \cdot \hat{n})(\vec{r} \times \hat{n})
$$

where $\vec{r}$ is the position vector of a surface element relative to a convenient origin, usually the center of mass. Note that we cannot use the area matrix here, since the cross-product must be taken inside the integral. From Eq. 78 we can make an order-of-magnitude estimate of the typical torque on 
an irregularly-shaped object, providing that all symmetry effects are disregarded. Approximating the moment of inertia by $m R^{2}$, where $R$ is a characteristic dimension of the object, we can write $\dot{\omega} \sim C_{m} I R / m$. Suppose $C_{m}=1$ dyne $\mathrm{W}^{-1}, I=100 \mathrm{~W} \mathrm{~cm}^{-2}$ (again, a time-averaged intensity), $R=10 \mathrm{~cm}$, and $m=100 \mathrm{~g}$. Then, after one second, the induced spin frequency is approximately $10 \mathrm{rad} \mathrm{s}^{-1}$. Of course, we cannot simply ignore torque-canceling symmetries, but this clearly shows that spin effects could play a role in laser/target interactions. A few examples are provided in the remainder of this section.

A second source of torque may arise if an object is already spinning, or if it is set to spinning as a result of asymmetry, as just discussed. In this case the ablation flow, viewed from the observer (inertial) frame is not purely normal with respect to a given surface, but contains both a normal component and an orthogonal $\vec{\omega} \times \vec{r}$ component. We refer to the torque imparted to the object from the effect of spin as the "kinematic torque," and symbolize it by $N_{k}$. To get an order-of-magnitude estimate of this effect, we take the azimuthal velocity component of the ablation flow to be $\sim \omega R$. The rate of angular momentum creation in the flow is therefore approximately $\omega R^{2}$ times the mass loss rate, which is $\sim C_{m} I R^{2} / v_{a}$ (with $v_{a}$ the ablation flow speed in the frame of the ablating surface), thus producing a back-reaction torque on the spinning object $N \sim C_{m} I R^{4} \omega / v_{a}$, with the result that $\dot{\omega} \sim$ $-\left(C_{m} I R^{2} / m v_{a}\right) \omega$, thus showing an exponential spin damping over the time scale $m v_{a} / C_{m} I R^{2}$. A more careful estimate would include the change to the moment of inertia from mass loss as ablation proceeds. In fact, as we show below, the mass loss effect on the moment of inertia may exactly compensate the torque, leaving the spin unaffected.

Formally, the kinematic torque can be calculated according to

$$
\vec{N}_{k}=\int d A \frac{d \dot{m}_{a}}{d A} \vec{r} \times(\vec{\omega} \times \vec{r})
$$

where we make the assumption that the local areal mass loss rate is given by

$$
\frac{d \dot{m}_{a}}{d A}=\frac{C_{m} I \hat{k} \cdot \hat{n}}{v_{a}}
$$

which is simply an expression of linear momentum conservation under the conditions that the ablation flow is parallel to the normal vector of the differential surface element in its rest frame, and that the momentum impulse 
is precisely anti-parallel to the surface normal. It can also be taken as a definition of the mechanical coupling coefficient $C_{m}$.

Given an inertia tensor $\mathbf{J}$, the torque equation (angular momentum transfer) is

$$
\frac{d}{d t}(\mathbf{J} \cdot \vec{\omega})=\vec{N}
$$

where the total torque is given by

$$
\vec{N}=\vec{N}_{s}+\vec{N}_{k}=C_{m} I \int d A \hat{k} \cdot \hat{n}\left[\vec{r} \times\left(\hat{n}+\frac{\vec{\omega} \times \vec{r}}{v_{a}}\right)\right]
$$

and we remind the reader that the integral is to be taken only over the illuminated portion of the target.

We work out a few idealized examples below. Ultimately, our goal is to determine the manner in which target spin might affect engagement strategies, if at all. Here, however, we focus on providing a few examples in the interest of imparting some sense of the scope of the problem.

\subsection{Mass Loss}

As discussed earlier, we follow the standard practice of ignoring mass-loss effects on the inertia of the debris target. We adopt the same procedure for dealing with spin changes induced by a static torque. However, in treating kinematic torque, we are required to treat the mass as a time-dependent quantity. These latter two choices are justified in this subsection.

For the static torque, a scalar approximation to Eq. 78 is

$$
\frac{d}{d t}(J \omega)_{S} \sim \dot{m}_{a} v_{a} R
$$

which bears a close resemblance to the linear momentum transfer equation

$$
\frac{d}{d t}(m v) \sim \dot{m}_{a} v_{a}
$$

Equation 83 can be thought of as leading to a "rotational rocket equation" when mass loss is treated explicitly. However, in the same way that mass is treated as a constant when working with linear momentum transfer through ablation, we assume a time-constant moment of inertia when working with rotation under the influence of a static torque, and obtain results to the same order of accuracy, i.e., the first-order correction term is $O\left(\Delta m / m_{o}\right)$. 
A similar scalar representation of the kinematic torque equation, from Eq. 79 , is

$$
\frac{d}{d t}(J \omega)_{K} \sim \dot{m}_{a} \omega R^{2}
$$

which expands to

$$
\dot{\omega} \sim \frac{\dot{m}_{a} R^{2}-\dot{J}}{J} \omega .
$$

The fraction on the right hand side of this equation is of the same order as $\dot{m}_{a} / m_{o}$, so that $\Delta \omega / \omega_{o} \propto \Delta m / m_{o}$ - rotation under the predominant influence of a kinematic torque demands an explicit accounting of the mass loss.

The relative magnitudes of the kinematic term and the static term are given by the ratio $\omega R / v_{a}$. Since this ratio is typically small compared to unity, the kinematic torque can, to first order, be ignored when a static torque is present. However, objects possessing a high degree of symmetry are often immune to static torques, whereas the kinematic torque is comparatively ubiquitous. In such cases it may be of interest to calculate the rotational dynamical effects of kinematic torque. We work through two examples below.

To summarize, working to leading order in $\Delta m / m$, we can treat the debris mass as a constant for the following two cases: (1) linear momentum transfer and (2) changes to rotation under the influence of a static torque. On the other hand, the mass should be treated in a time-dependent manner when evaluating rotational changes under the sole influence of a kinematic torque.

\subsection{Kinematic Torque on a Spinning Cylinder}

An example of kinematic torque is provided by a cylinder of radius $r_{c}$ and height $h$, spinning on its main axis, and illuminated only on its curved surface, as illustrated in Fig. 9. From Eq. 78 it is easy to show that the static torque term vanishes.

We need the following vectors:

$$
\hat{k}=\hat{y} \quad \vec{\omega}=\omega \hat{z} \quad \hat{n}=\left(\begin{array}{c}
\cos \phi \\
\sin \phi \\
0
\end{array}\right) \quad \vec{r}=\left(\begin{array}{c}
r_{c} \cos \phi \\
r_{c} \sin \phi \\
z
\end{array}\right)
$$

where $\phi$ is the standard azimuthal angle measuring angular displacement

with respect to the positive $x$-axis. Given the initial condition, only the $z$-component is relevant. Therefore, we will need to solve

$$
\dot{J}_{z z} \omega+J_{z z} \dot{\omega}=N_{z}
$$



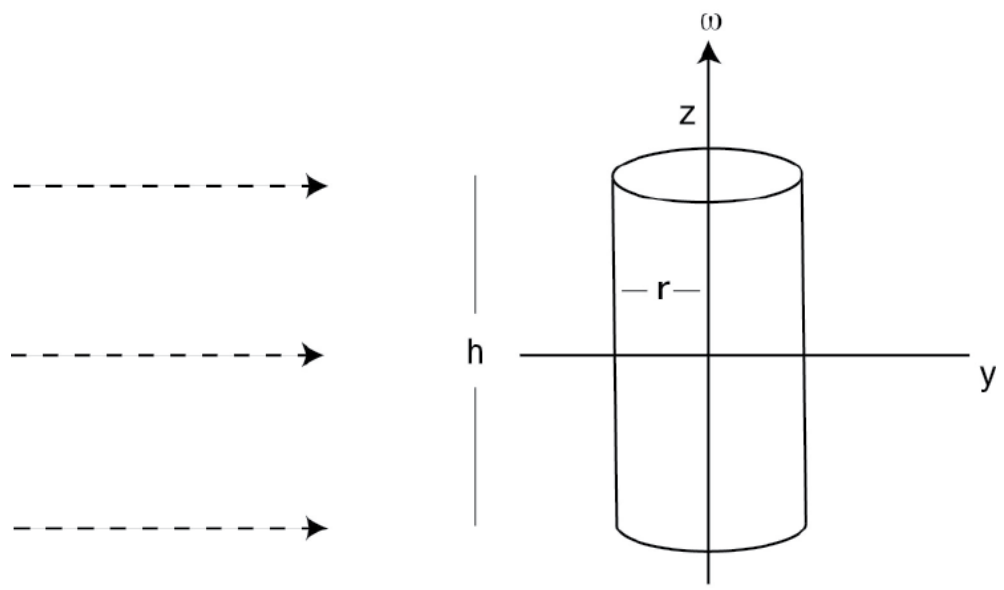

Figure 9: Spinning cylinder of height $h$ and radius $r$, illuminated from the left (dashed arrows), with the laser propagation vector $(\hat{k}=\hat{y})$ perpendicular to the spin axis $(\vec{\omega}=\omega \hat{z})$.

where $J_{z z} \equiv J=(1 / 2) M r_{c}^{2}$. Eliminating $r_{c}$ according to $r_{c}=(M / \pi \rho h)^{1 / 2}$, we have $J=M^{2} / 2 \pi \rho h$ and $\dot{J}=M \dot{M} / \pi \rho h$, which allows us to write Eq. 88 as

$$
\frac{M \dot{M}}{\pi \rho h} \omega+\frac{M^{2}}{2 \pi \rho h} \dot{\omega}=N_{z}
$$

We let both $M$ and $\dot{M}$ be functions of time, accounting explicitly for the reduction of mass and the shrinking target cross-section as ablation proceeds.

The mass loss rate is found from Eq. 80, which takes the form

$$
\dot{M}=\frac{C_{m} I h r_{c}(t)}{v_{a}} \int_{\pi}^{2 \pi} d \phi \sin \phi=-\frac{2 C_{m} I h r_{c}(t)}{v_{a}} .
$$

We allow for mass loss to reduce only the radius of the cylinder, hence $r_{c} \rightarrow$ $r_{c}(t)$, but assume that $h$ is a constant, i.e., no endcap illumination for this example.

By again re-expressing $r_{c}$ in terms of $M$, Eq. 90 becomes

$$
\dot{M}=-\frac{2 C_{m} I h}{v_{a}}\left(\frac{M}{\pi \rho h}\right)^{1 / 2}
$$

from which

$$
M=M_{o}(1-\beta t)^{2} \quad \dot{M}=-2 M_{o} \beta(1-\beta t)
$$


for an initial mass $M_{o}$, and where we define $\beta$, with dimension of inverse time, according to

$$
\beta \equiv \frac{C_{m} I}{v_{a}}\left(\frac{h}{\pi \rho M_{o}}\right)^{1 / 2} .
$$

Next, to find the torque acting on the cylinder, we use Eq. 82, which, recalling that the static torque is zero, becomes

$$
\vec{N}=\frac{C_{m} I}{v_{a}} \int d A \hat{k} \cdot \hat{n}\left[r^{2} \vec{\omega}-(\vec{\omega} \cdot \vec{r}) \vec{r}\right]
$$

(using the BACCAB rule for the triple vector product, and also noting that $\left.r^{2}=r_{c}^{2}+z^{2}\right)$ with the result

$$
N_{z}=-\frac{2 M_{o}^{2} \beta}{\pi \rho h}(1-\beta t)^{3} \omega
$$

The torque, as expected, depletes the cylinder of angular momentum, while the magnitude of the torque decreases with time, since the target crosssection is shrinking.

Finally, to find the net effect on the spin, we combine Eqs. 89, 92, and 95 to give

$$
-\frac{2 M_{o}^{2} \beta}{\pi \rho h}(1-\beta t)^{3} \omega+\frac{M_{o}^{2}}{2 \pi \rho h}(1-\beta t)^{4} \dot{\omega}=-\frac{2 M_{o}^{2} \beta}{\pi \rho h}(1-\beta t)^{3} \omega .
$$

Since the first term on the left-hand-side of Eq. 96 (reduction of the moment of inertia through mass loss) entirely accommodates the torque (right-hand side), we find $\dot{\omega}=0$, i.e., the spin is unaffected by laser irradiation in this configuration. In short, the cylinder's loss of angular momentum through mass loss is sufficient to balance the torque acting on the cylinder. To take another example, although somewhat more involved, it is straightforward to show that the same effect holds for a right circular cone spinning about its main axis, if illuminated down the axis.

\subsection{Kinematic Torque on a Spinning Sphere}

We showed in the previous example that laser irradiation does not add or subtract spin to a side-illuminated spinning cylinder. In this subsection, we replace the cylinder with a uniform sphere of radius $R$, with the spin axis again aligned with the $z$-axis, and the laser propagating along the positive 
$y$-axis (equatorial illumination), then perform the same calculation (see Fig. 9 for reference).

The mass and mass loss rate as functions of time are derived in a manner similar to the previous section, i.e., using $J=(2 / 5) M R^{2}$ and $R=$ $(3 M / 4 \pi \rho)^{1 / 3}$, with the results

$$
M=M_{o}\left(1-\beta_{s} t\right)^{3} \quad \dot{M}=-3 M_{o} \beta_{s}\left(1-\beta_{s} t\right)^{2}
$$

where $\beta_{s}$ is analogous to the decay constant $\beta$ used for the cylinder in the previous section;

$$
\beta_{s}=\frac{C_{m} I}{2 v_{a}}\left(\frac{\pi}{6 \rho^{2} M_{o}}\right)^{1 / 3}
$$

Then, using the expressions for $M$ and $\dot{M}$ from Eq. 97,

$$
\frac{d}{d t}(J \omega)=-2\left(\frac{3}{4 \pi \rho}\right)^{2 / 3} M_{o}^{5 / 3}\left(1-\beta_{s} t\right)^{4}\left[\beta_{s} \omega-\frac{1}{5}\left(1-\beta_{s} t\right) \dot{\omega}\right] .
$$

From Eq. 82, again discarding the static term, the torque on the sphere is

$$
N_{z}=-\frac{9}{4}\left(\frac{3}{4 \pi \rho}\right)^{2 / 3} M_{o}^{5 / 3} \beta_{s}\left(1-\beta_{s} t\right)^{4} \omega
$$

Equating the right-hand side of Eq. 99 to the right-hand side Eq. 100, canceling common factors, gives

$$
-2 \beta_{s} \omega+\frac{2}{5}\left(1-\beta_{s} t\right) \dot{\omega}=-\frac{9}{4} \beta_{s} \omega
$$

whose solution is

$$
\omega=\omega_{o}\left(1-\beta_{s} t\right)^{5 / 8}
$$

Thus the laser will cause the sphere to spin down. The mathematical solution is only an approximation, of course, since the target will not maintain a perfectly spherical shape, given that the equator loses mass more readily than the polar regions in this orientation.

\subsection{Asymmetric Dumbbell: An Ablation-Driven Pendulum}

Referring to Fig. 10, consider two spherical masses, with radii $R_{1}$ and $R_{2}$ $\left(R_{1}>R_{2}\right)$, identical mass density $\rho$ (so that $\left.M_{1}>M_{2}\right)$, positioned at $\vec{r}_{1}$ and $\vec{r}_{2}$, connected by a massless rod, with the separation of the centers of the spheres $r_{1}+r_{2} \equiv a$, and irradiated such that $\hat{k}=-\hat{x}$. To keep things simple, 


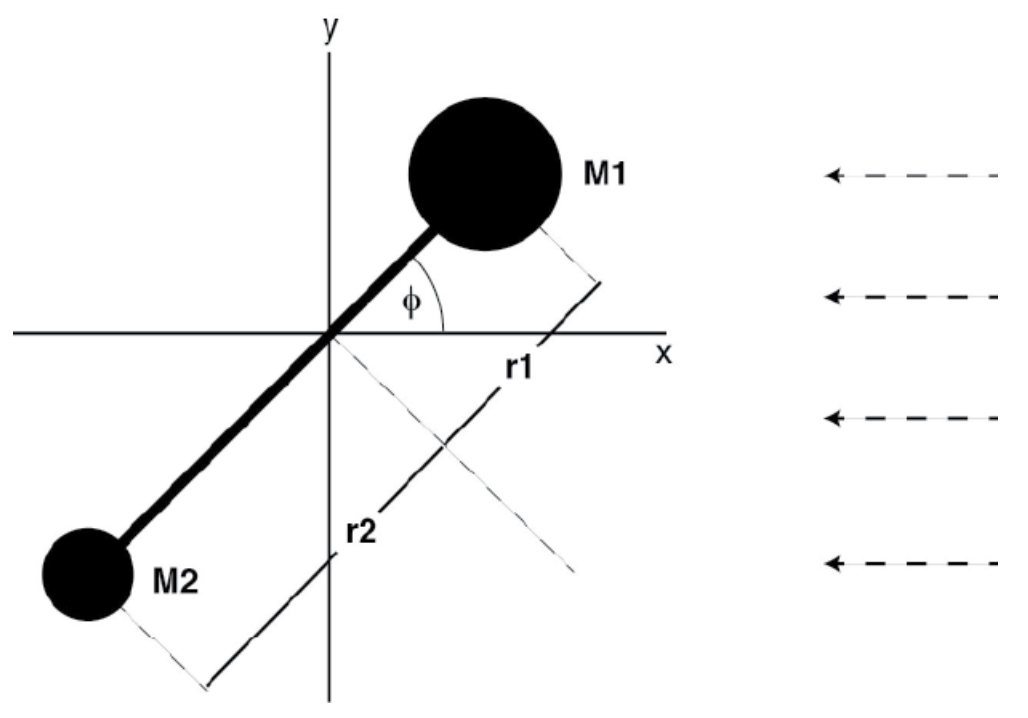

Figure 10: Geometric relations for an asymmetric dumbbell consisting of two spheres of identical material but different radii $\left(R_{1}>R_{2}\right.$, hence $\left.M_{1}>M_{2}\right)$. The rotation vector lies along the $z$-axis, with rotation about the system center of mass, which coincides with the origin. The angular position is described by the angle $\phi$, as shown. Laser is incident from the right (dashed arrows).

we make the assumption that each sphere has a radius substantially smaller than the separation distance of the two spheres, so that the scalar moment of inertia is simply $J=\mu a^{2}$ for the reduced mass $\mu$. We ignore the change to the moment of inertia caused by mass loss as per the discussion in $§ 5.1$. Also, we ignore the shadowing of one sphere by the other.

With the approximation that the static torque applies only to the spheres, and not to the rod, we have

$$
\vec{N}_{s}=C_{m} I \sum_{\alpha} \int d A_{\alpha}\left(\hat{k} \cdot \hat{n}_{\alpha}\right)\left(\vec{r}_{\alpha} \times \hat{n}_{\alpha}\right),
$$

where $\alpha$ subscripts the two spheres 1 and 2. Relying on our approximation that $R_{\alpha}$ is small compared to $r_{\alpha}$, this can be approximated by

$$
\vec{N}_{s}=C_{m} I \sum_{\alpha} \vec{r}_{\alpha} \times\left(\hat{k} \cdot \mathbf{G}_{\alpha}\right)=\frac{2}{3} \pi C_{m} I \sum_{\alpha} R_{\alpha}^{2} \vec{r}_{\alpha} \times \hat{k},
$$

where $\mathbf{G}_{\alpha}=(2 \pi / 3) R_{\alpha}^{2} \mathbf{I}$, using our result from $\S 4.2$. The torque has only a 
$z$-component, which is, after evaluating the previous expression,

$$
N_{z}=-\frac{2}{3} \pi C_{m} I\left(r_{2} R_{2}^{2}-r_{1} R_{1}^{2}\right) \sin \phi .
$$

Using $R_{\alpha}=\left(3 m_{\alpha} / 4 \pi \rho\right)^{1 / 3}, r_{1}=\left(m_{2} / m\right) a$, and $r_{2}=\left(m_{1} / m\right) a$, the equation of motion becomes

$$
\ddot{\phi}+\Omega^{2} \sin \phi=0,
$$

where

$$
\Omega^{2}=\left(\frac{\pi}{6 \rho^{2}}\right)^{1 / 3} \frac{C_{m} I}{a}\left(\frac{1}{m_{2}^{1 / 3}}-\frac{1}{m_{1}^{1 / 3}}\right),
$$

which is mathematically identical to the non-linear pendulum equation.

While the governing equation of motion is the same as for a pendulum in a uniform gravitational field, the analogy is not perfect. For one thing, if our two spherical masses were connected by a rod of negligible mass, and allowed to rotate about the center of mass in a gravitational field, there would be no rotation other than that imparted by some other means, since the gravitational torques would precisely cancel each other. Also, the "natural frequency" $\Omega$ is not an intrinsic property of the dumbbell configuration - it is not "natural" at all - since it scales with $C_{m}^{1 / 2}$, and has a $\rho$-dependence, as well, whereas the motion of a pendulum is not related to its composition in any way. However, we note that the linear scale $a$ plays the same role as the length of a pendulum arm, and, crudely speaking, the quantity $C_{m} I /\left(\rho^{2} m\right)^{1 / 3}$ is analogous (and dimensionally equivalent) to the gravitational acceleration.

Equation 106 can be rewritten as

$$
\frac{d}{d t}\left(\frac{1}{2} \dot{\phi}^{2}-\Omega^{2} \cos \phi\right)=0,
$$

which, with initial conditions, gives $\omega(\phi)(\omega \equiv \dot{\phi})$.

$$
\omega^{2}=\omega_{o}^{2}+2 \Omega^{2}\left(\cos \phi-\cos \phi_{o}\right) .
$$

If the system is initially stationary $\left(\omega_{o}=0\right)$, rotational motion starts at the moment ablation begins - $\Omega$ is "switched on" — with the angular velocity

$$
\omega^{2}=2 \Omega^{2}\left(\cos \phi-\cos \phi_{o}\right) .
$$

The angular range is restricted to $\left[-\phi_{o}, \phi_{o}\right]$ - the dumbbell "swings" with an amplitude-dependent period $P$, which can be expressed in terms of 
the complete elliptical integral of the first kind $K$ (Belendez et al., 2007), according to

$$
P=\frac{4}{\Omega} K\left[\sin ^{2}\left(\phi_{o} / 2\right)\right] .
$$

Using the series expansion for $K$ (Abramowitz and Stegun, 1972)

$$
K\left[\sin ^{2}\left(\phi_{o} / 2\right)\right]=\frac{\pi}{2}\left[1+\frac{1}{4} \sin ^{2} \frac{\phi_{o}}{2}+O\left(\sin \left(\phi_{o} / 2\right)\right)^{4}\right],
$$

and retaining only the first term in the limit of small $\phi_{o}$, the period for a simple harmonic oscillator $2 \pi / \Omega$ is recovered. Of course, this also follows from the direct solution of Eq. 106 in the limit $\sin \phi \rightarrow \phi$.

If $\omega_{o}$ is non-zero, there is the possibility that the system will not reverse itself but continue its rotational motion, albeit in a complex way compared to its initial uniform rotation. This depends on the initial condition pair $\left(\omega_{o}, \phi_{o}\right)$. Specifically, rotation is maintained if

$$
\omega_{o}^{2}>2 \Omega^{2}\left(1+\cos \phi_{o}\right) .
$$

The analogy to a nonlinear pendulum still holds; in this case the initial condition is analogous to providing a starting push to the pendulum. If its total energy at that instant exceeds the potential energy at the top of its circular trajectory, it will "loop the loop," and continue on, absent rotational damping.

\subsection{Wedge}

We now consider two identical rectangular plates, joined to form a wedge. The problem of a wedge is complicated by self-shadowing over a certain range of orientations relative to the laser beam. In the interest of simplicity, we will restrict the possible range of orientations so that self-shadowing does not occur. We use the coordinates and set the wedge characteristics in accordance with Fig. 11. The angle $\gamma$ is the half-angle separation between the two plates, which, for simplicity, we assume to be of equal area $(A / 2)$. The approach here works equally well if the plates are of unequal area, but we work with the simpler example here. We define a length and width, $L$ and $h$, respectively, such that $A / 2=L h$. If the wedge is oriented such that a line that bisects the angle between the wedge faces is parallel to the laser propagation vector (along the $y$-axis), then, as we show below, this is an equilibrium position as regards spin. Therefore, we define an angle $\phi$ to quantify angular deviations 


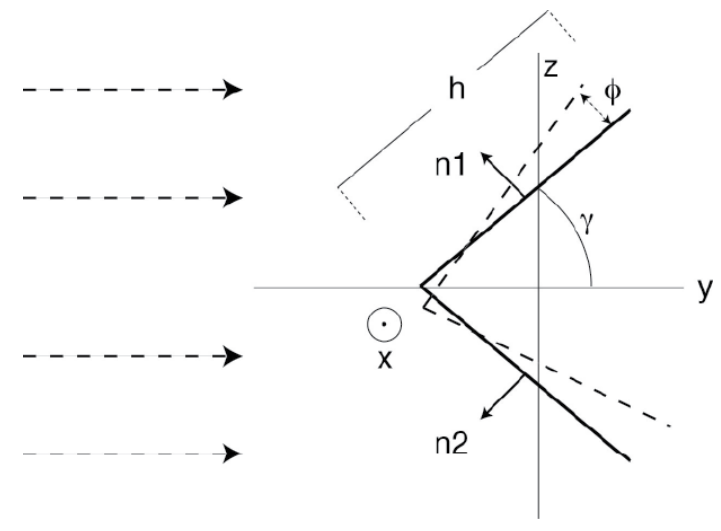

Figure 11: Diagram of a wedge composed of two thin rectangular plates of equal area ( $h \times L ; h$ shown, $L$, the $x$ dimension, not shown), of arbitrary opening angle $2 \gamma$ with the line of intersection parallel to the $x$-axis. Origin coincides with the center of mass. Translational motion is restricted to the $y z$-plane, and rotation about the center of mass is parallel to the $x$-axis. Laser irradiation is from the left (dashed arrows), and the two exposed surface normals $\hat{n}_{1}$ and $\hat{n}_{2}$ are indicated. The angle $\phi$ tracks a small angular displacement (dashed lines) from the symmetric, torque-free orientation (heavy lines).

from this equilibrium. The no-self-shadowing requirement demands only that $\phi \leq \gamma$

With the two surface normals given by

$$
\hat{n}_{1}=\left(\begin{array}{c}
0 \\
-\sin (\gamma+\phi) \\
\cos (\gamma+\phi)
\end{array}\right) \quad \hat{n}_{2}=\left(\begin{array}{c}
0 \\
-\sin (\gamma-\phi) \\
-\cos (\gamma-\phi)
\end{array}\right)
$$

the area matrix becomes

$$
\mathbf{G}=\frac{A}{2}\left(\begin{array}{ccc}
0 & 0 & 0 \\
0 & 1-\cos 2 \gamma \cos 2 \phi & -\cos 2 \gamma \sin 2 \phi \\
0 & -\cos 2 \gamma \sin 2 \phi & 1+\cos 2 \gamma \cos 2 \phi
\end{array}\right)
$$

and, with $\hat{k}=\hat{y}$, the force equation for the center of mass is

$$
m \frac{d \vec{v}}{d t}=\frac{1}{2} C_{m} I A\left(\begin{array}{c}
0 \\
1-\cos 2 \gamma \cos 2 \phi \\
-\cos 2 \gamma \sin 2 \phi
\end{array}\right)
$$




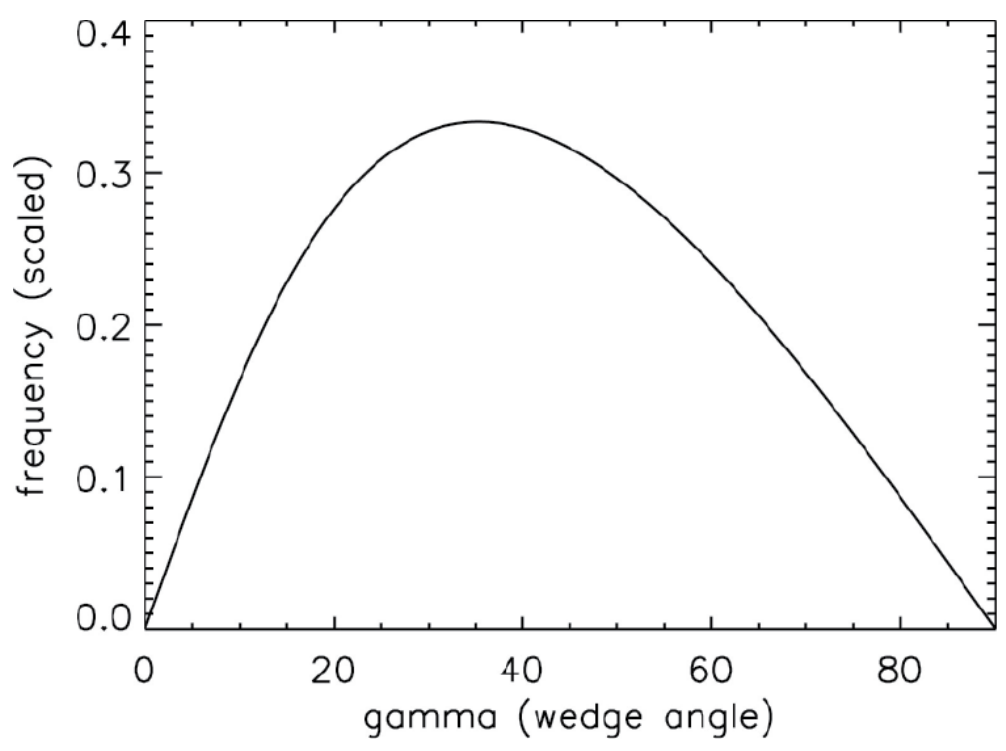

Figure 12: Spin-dependent frequency of an irradiated wedge vs. the wedge half-opening angle $\gamma$ defined by Fig. 11. The frequency is scaled as a multiple of $\left(6 C_{m} I / \sigma h\right)^{1 / 2}$ (see text).

There are two special cases worth noting. If $\phi=0$, there is no $z$-component to the impulse. The $y$-component becomes

$$
m \dot{v}_{y}=C_{m} I A \sin ^{2} \gamma,
$$

which, by symmetry, shows the expected similarity to the case of an arbitrarily oriented flat plate (see Eq. 44). For non-zero $\phi$, but $\gamma=\pi / 4$ (right-angle wedge), the $z$-component of acceleration again vanishes, and the $y$-component becomes invariant to orientation, both features being analogous to the case of a cube, as discussed in $\S 4.1$.

The moment of inertia for rotation about the center of mass, such that the angular velocity vector is parallel to the $x$-axis, is

$$
J_{x x}=\frac{1}{12} m h^{2}\left(1+3 \sin ^{2} \gamma\right) .
$$

We eliminate the mass in favor of a surface mass density $\sigma$ (e.g., with dimension $\mathrm{g} \mathrm{cm}^{-2}$ ), such that $m=2 \sigma L h$, which gives from the torque equation

$$
\ddot{\phi}=-\frac{6 C_{m} I}{\sigma h} \frac{\cos ^{2} \gamma \sin ^{2} \gamma}{1+3 \sin ^{2} \gamma} \sin \phi,
$$


which, as was the case for an asymmetric dumbbell, is the equation of an undamped nonlinear pendulum. In the limit of small $\phi$, the system behaves like a simple harmonic oscillator with a "natural" frequency given by

$$
\Omega^{2}=\frac{6 C_{m} I}{\sigma h} \frac{\cos ^{2} \gamma \sin ^{2} \gamma}{1+3 \sin ^{2} \gamma},
$$

which attains a maximum value of $\Omega_{\max }^{2}=2 C_{m} I / 3 \sigma h$, when the wedge opening half-angle $\gamma=\arcsin 3^{-1 / 2}$. The quantity $\left(\sigma h / 6 C_{m} I\right)^{1 / 2} \Omega$ (a dimensionless shape-dependent frequency) is plotted against $\gamma$ in Fig. 12.

\subsection{Cone}

For our final example, consider a right circular cone, of uniform density $\rho$ and mass $m$, with opening half-angle $\alpha$, such that $\tan \alpha=R / H$, where $R$ is the base radius and $H$ is the height, as illustrated in Fig. 13. We specialize the treatment here to cases for which self-shadowing is not present, which means that the beam vector is restricted to lie within a conical region delineated by the dashed lines in Fig. 13. The more complicated case of self-shadowing can be worked out analytically, but is somewhat tedious, so we choose to look only at the simpler case here.

The coordinate system is shown in the lower right-hand side of Fig. 13, with the positive $x$-axis pointing out of the page. If the angle $\phi$ corresponds to the azimuthal angle as defined for standard spherical coordinate systems, then the surface normal is given by

$$
\hat{n}=\left(\begin{array}{c}
\cos \alpha \cos \phi \\
\cos \alpha \sin \phi \\
\sin \alpha
\end{array}\right) .
$$

If we let $\eta \equiv H / R=\cot \alpha$, then the area matrix is

$$
\mathbf{G}=\frac{\pi R^{2}}{\left(1+\eta^{2}\right)^{1 / 2}}\left(\begin{array}{ccc}
\eta^{2} / 2 & 0 & 0 \\
0 & \eta^{2} / 2 & 0 \\
0 & 0 & 1
\end{array}\right)
$$

We see immediately that for the special case $H=R \sqrt{2}$, the area matrix is proportional to the unit tensor, and the momentum response is precisely parallel to the beam. For all other cases, a perpendicular component will be present. 

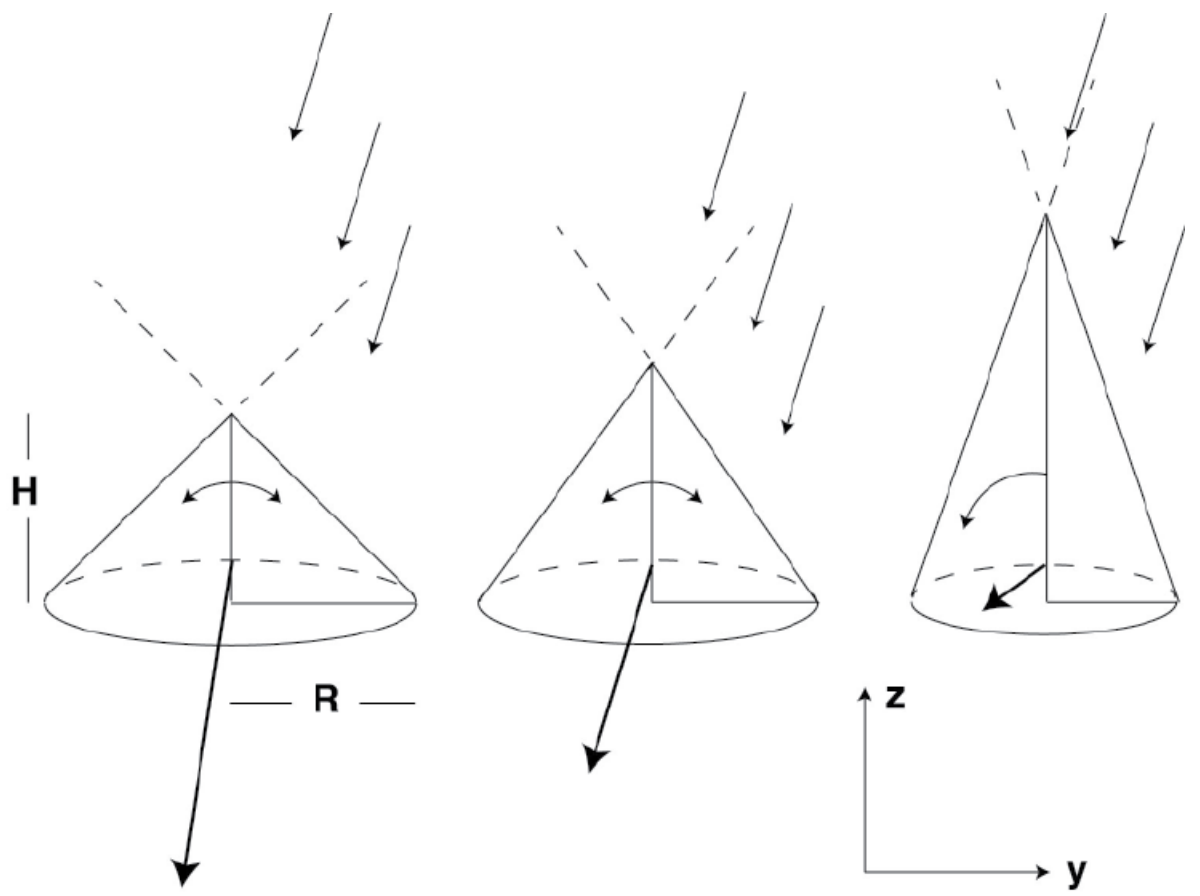

Figure 13: Three laser-irradiated cones of equal mass with different values of $H / R ; 1.0$ (left); $\sqrt{2}$ (center); 3.0 (right). The laser propagation vector $\hat{k}$ lies in the $y z$-plane, tilted $17^{\circ}$ off the $z$-axis (triple arrow pattern at top of each cone). The acceleration vectors at ablation onset are shown (heavy arrows), each lying in the $y z$-plane, with the directions and relative magnitudes represented quantitatively. For $H / R=\sqrt{2}$ (center) the impulse is parallel to the beam, independent of the laser orientation, while for smaller (larger) values, the impulse is nearer (farther from) the axis. The back-and-forth arrowed arcs denote pendulum-type oscillations, while the leftward arc on the right-side cone indicates "tipping." For $H / R=2 \sqrt{2}$ (not shown), the cone is stable against torques, and does not oscillate, also independent of the laser orientation. 
The laser propagation vector is described by its angular displacement $\psi$ relative to the axis of the cone;

$$
\hat{k}=\left(\begin{array}{c}
0 \\
-\sin \psi \\
-\cos \psi
\end{array}\right)
$$

so that

$$
\hat{k} \cdot \hat{n}=-\cos \alpha \sin \phi \sin \psi-\sin \alpha \cos \psi,
$$

with the restriction $\psi \leq \alpha$ satisfying the no-self-shadowing provision. We note that the illumination condition $\hat{k} \cdot \hat{n}<0$ is, from the previous equation, equivalent to

$$
\sin \phi>-\tan \alpha \cot \psi
$$

which sets the range of $\phi$-integration for the more general case.

The equation of motion becomes

$$
\frac{d \vec{v}}{d t}=-\frac{C_{m} I}{\left(1+\eta^{2}\right)^{1 / 2}}\left(\frac{9 \pi}{\eta^{2} \rho^{2} m}\right)^{1 / 3}\left(\begin{array}{c}
0 \\
\left(\eta^{2} / 2\right) \sin \psi \\
\cos \psi
\end{array}\right)
$$

showing that the relative parallel and perpendicular impulse components depend on both the illumination angle and the shape of the cone. We leave the topic of translational motion of the cone here, and move on to rotational motion.

For the following calculations, we place the origin at the center of mass. Points on the conical surface can be specified by $z$ and $\phi$, such that

$$
\vec{r}=\left(\begin{array}{c}
f(z) \cos \phi \\
f(z) \sin \phi \\
z
\end{array}\right)
$$

where $f(z)=(3 / 4) R-(R / H) z$. The domain of $z$ is $[-H / 4,3 H / 4]$, and for the integration of Eq. 78, we have $d A=f(z) d \phi d z / \cos \alpha$.

Only the $x$-component of the cross-product in Eq. 78 leads to a nonvanishing torque component;

$$
(\vec{r} \times \hat{n})_{x}=f(z) \sin \alpha \sin \phi-z \cos \alpha \sin \phi .
$$




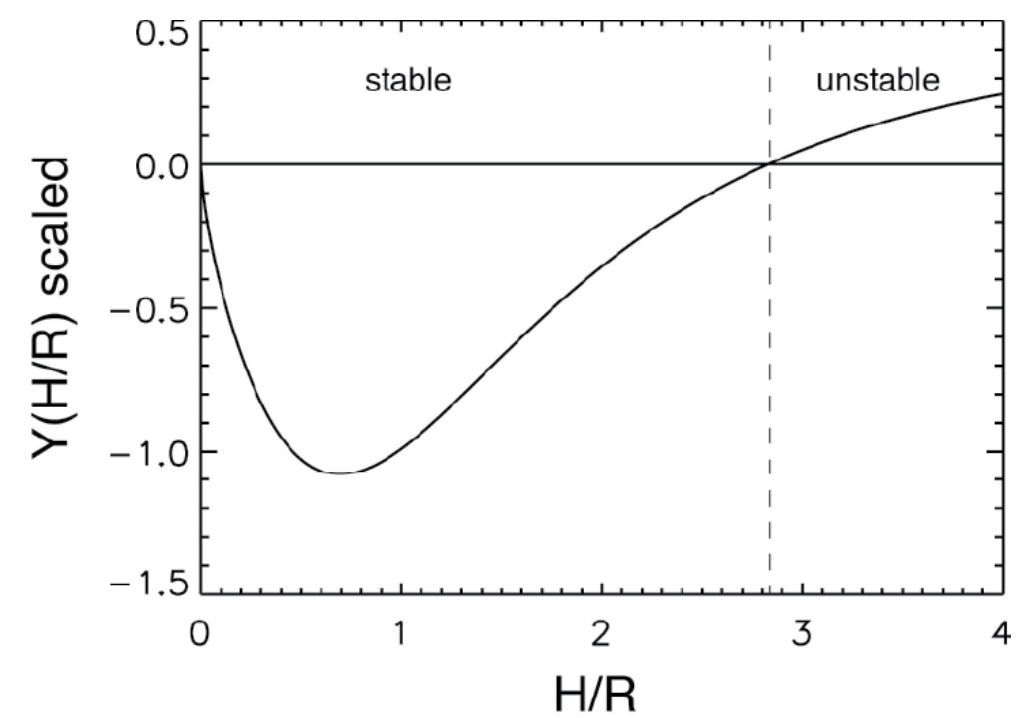

Figure 14: The $\eta$-dependent portion of the function $Y(\eta)(\eta \equiv H / R$; see Eq. 133), describing the rotational response of a cone to laser ablation. The vertical dashed line delineates regions of stable nonlinear pendulum rotation (left side), and "runaway tipping" (right side).

After multiplying Eq. 128 by $\hat{k} \cdot \hat{n}$, and eliminating terms that vanish upon $\phi$-integration, we are left with

$$
N_{x}=-C_{m} I \sin \psi \int_{0}^{2 \pi} d \phi \sin ^{2} \phi \int_{-H / 4}^{3 H / 4} d z f(z)[f(z) \sin \alpha-z \cos \alpha],
$$

which leads to

$$
N_{x}=-\frac{C_{m} I m}{\rho} \frac{1-\eta^{2} / 8}{\left(1+\eta^{2}\right)^{1 / 2}} \sin \psi .
$$

The torque can be positive, negative, or zero depending on the choice of $\eta$.

The moment of inertia corresponding to $J_{x x}$ for rotation about the center of mass is

$$
J_{x x}=\frac{3}{20} m R^{2}\left(1+\frac{1}{4} \eta^{2}\right)=\frac{3}{20}\left(\frac{3}{\pi \rho \eta}\right)^{2 / 3}\left(1+\frac{1}{4} \eta^{2}\right) m^{5 / 3}
$$

Therefore, after some re-arrangement, the equation of rotational motion becomes

$$
\ddot{\psi}=Y(\eta) \sin \psi,
$$


with $Y(\eta)$ given by

$$
Y(\eta)=\frac{10}{3}\left(\frac{\pi^{2}}{9 \rho m^{2}}\right)^{1 / 3} C_{m} I\left[\frac{\eta^{2 / 3}}{\sqrt{1+\eta^{2}}} \frac{\eta^{2}-8}{\eta^{2}+4}\right]
$$

Since $Y$ can be positive, negative, or zero, there are three types of rotational behavior, pendulum-type oscillation for cones with $H<2 R \sqrt{2}$, unstable "tipping" for $H>2 R \sqrt{2}$, and metastability against rotation for $H=2 R \sqrt{2}$. The bracketed factor in Eq. 133 is plotted in Fig. 14.

\section{Discussion}

Our goals here are: (1) to provide the means by which to adjust or augment some of the intuitive notions that have been built upon one-dimensional modeling and experiments that involve flat plates, and (2) to provide a framework for carrying out more elaborate orbital calculations than what are required under the assumption that potential debris targets are spheres or ideally aligned flat plates. Still, even beyond technological questions related to our ability to accurately track and engage small debris from a ground station [see discussion in Phipps et al. (2012) and references therein], a capability which is pre-supposed here, there are a number of additional refinements that are likely necessary to further quantify the response of an object to laser ablation, as discussed in the remainder of this section.

We can look at laser-induced shape deformation in two ways: (1) smooth, surface-wide deformation, and (2) creation of local smaller scale irregularities. The first category might be exemplified by the problem treated in $\S 5.3$, a rotating sphere subjected to ablation that is inherently non-uniform, owing simply to the curvature of the surface. The second category includes intrinsic surface irregularities that change the local intensity on target relative to the perfect surfaces we have assumed (a $\hat{k} \cdot \hat{n}$ effect), as well as irregularities that may be created by beam imperfections, particularly those caused by atmospheric propagation. Although adaptive optics techniques mitigate this latter effect, deviations from perfection are not unlikely. Spatial beam inhomogeneities result in variations in the local mass loss rate, which leads to non-uniform surface deformation. Moreover, the presence of local variations in the incident intensity means that, strictly speaking, the coupling itself varies locally, irrespective of surface irregularities. But it is worth noting that if small-scale irregularities tend to reduce the incident energy deposition 
rate via the $\hat{k} \cdot \hat{n}$ dependence, such reductions tend to improve the coupling for the operating range of interest, partially offsetting the pure intensity dependence of the local mass loss rate. Progressive surface deformation and its effect on momentum transfer could be included in more exacting numerical work. No doubt, laboratory measurements of the evolution of a target subjected to multiple ablation events would prove useful.

We noted in $\S 2.2$ our assumption that $C_{m}$ is treated in this paper as a constant across the illuminated surface of a given object. While we argued for the validity of this approximation, a suggested improvement for future work is to account explicitly for the intensity dependence of the coupling: $C_{m} \rightarrow C_{m}(I)$. Again, the sparseness of the experimental database becomes a factor in accurately invoking this functional dependence. We stress that the quantities of primary interest to debris clearing are directly proportional to the magnitude of the coupling coefficient (recoil velocities, spin damping constants), or to its square root (induced spin frequencies). Accurate numerical predictions of momentum transfer presuppose an adequately comprehensive and experimentally precise database of coupling coefficients.

A potential concern to debris clearing programs can be expressed in terms of the well-known maxim of medical ethics: "first, do no harm"; one must ask whether or not laser ablation will simply exacerbate the problem by creating many fragments from one. The target recoil during an ablation event creates a pressure pulse in the target material. Experimental data can be approximated by the expression (Phipps et al., 1988; Fabbro et al., 1990)

$$
P=3.9 I^{0.7} \lambda^{-0.3} \tau^{-0.15} \text { kbars, }
$$

where the intensity, laser wavelength, and pulse duration are expressed as multiples of $\mathrm{GW} \mathrm{cm}^{-2}, \mu \mathrm{m}$, and ns, respectively. (Some care with Eq. 134 should be exercised, since it describes a trend line only.) The response of a debris object to a pressure pulse is highly material-dependent. It is not unlikely that some spallation may occur (Zeldovich and Raizer, 2002). The spall thickness - the characteristic size of potential spallation fragments - is roughly the material sound speed times the pulse duration. For sound speeds of a few times $10^{5} \mathrm{~cm} \mathrm{~s}^{-1}$, and a pulse duration of a few nanoseconds, the spall thickness $\sim 10 \mu$. Although these spallation products would not pose a direct threat to space assets, the subsequent laser/target momentum coupling may be affected by the resulting shape modification. Laboratory studies in the ns-pulse $10^{8}-10^{10} \mathrm{~W} \mathrm{~cm}^{-2}$ regime of progressive target deformation caused 
by the combination of ablation and possible spallation events are desirable in order to identify the prevalence and overall nature of this type of shape evolution.

Finally, we note that our goal (2) above is only partially addressed here. We have said nothing about the consequences for calculations of orbital modifications, which are essential to planning sustained laser engagements, target re-acquisition when multiple-orbit engagements are needed, and ultimately, optimizing the perigee reduction per unit laser energy expended. We have shown that targets will typically acquire off-beam impulse components. Calculations of orbital angular momentum transfer and energy transfer either to or from a debris fragment must include a new set of vectors, namely, those that describe the illuminated target surfaces. In terms of engaging spinning targets, we have shown that assuming a net impulse along the beam by spin-averaging does not suffice to predict resultant orbits, and that off-beam deflections depend on the spin state of the target at the onset of ablation. Also, target spin cannot be de-coupled from the linear momentum transfer calculations, since the area matrix becomes time-dependent in that case. In that sense, the efficiency of linear momentum transfer is also dependent upon the initial spin state. In future work, we will begin to examine the consequent effects on orbital trajectories in low-Earth orbit.

The relations among the laser station coordinates, orbital parameters, and selection of laser engagement time intervals are made more complex by having to deal with shape effects. However, these complications are manageable, as shown by our analyses. In fact, they can be used to help refine predictive simulations of debris de-orbiting campaigns, enabling more precise descriptions of the behavior of the debris population in response to laser ablation. While the calculations in this work have been carried out for a few idealized shapes, our methods can be used with 3-D models of real objects, invoking a variety of materials and shapes, to develop a comprehensive modeling package for pulsed laser de-orbiting or deflection of space debris.

\section{Acknowledgements}

The authors thank the referees for providing useful suggestions that have helped to improve the clarity of the manuscript. Lawrence Livermore National Laboratory is operated by Lawrence Livermore National Security, LLC, for the U.S. Department of Energy, National Nuclear Security Administration under Contract DE-AC52-07NA27344. 


\section{References}

Abramowitz, M., and Stegun, I.A. Handbook of Mathematical Functions. Dover Publications, Inc., New York, 1972.

Bate, R.R., Mueller, D.D., White, J.E. Fundamentals of Astrodynamics. Dover Publications, Inc., New York, 1971.

Beckers, J. Adaptive optics for astronomy - Principles, performance and applications. In: Annual Review of Astronomy and Astrophysics, 31, A9412726 02-90, G. Burbidge, G. (ed.), 13-62, 1993.

Belendez, A., Pascual, C., Mendez, D.I., et al. Exact solution for the nonlinear pendulum. Revista Brasileira de Ensino de Fisica, 29, 4, 2007.

Campbell, J.W. ORION. Laser and Particle Beams, 14, 1, 1-44, 1996a.

Campbell, J.W. Project ORION: Orbital Debris Removal Using GroundBased Sensors and Lasers. J.W. Campbell, ed., NASA Marshall Spaceflight Center Technical Memorandum 108522, $1996 \mathrm{~b}$.

Dunne, A.M., et al. Timely delivery of laser inertial fusion energy (LIFE). Fusion Science and Technology 60, 19-27 2011.

Fabbro, R., Fournier, J., Ballard, P., et al. Physical study of laser-produced plasma in confined geometry. J. Appl. Phys., 68, 775, 1990.

Goldstein, H. Classical Mechanics. Second Edition, Addison-Wesley Publishing Company, 145-147, 1980.

Kessler, D.J., and Cour-Palais, B.G. Collision frequency of artificial satellites: The creation of a debris belt. J. Geophys. Res. 83, A6, 1978.

Kessler, D.J. in: Orbital Debris from Upper-Stage Breakup. Progress in Astronautics and Aeronautics, 121, 3, 1989.

Kidder, R.E. in Physics of High Energy Density, Proceedings of the International School of Physics Enrico Fermi (Academic Press: New York and London), 306, 1971.

Klinkrad, H. Space Debris, Models and Risk Analysis. Springer/Praxis Publishing, Chichester UK, 2006. 
Liedahl, D.A., Libby, S.B., Rubenchik, A. Momentum transfer by laser ablation of irregularly shaped space debris. AIP Conference Proceedings, 1278, $772,2010$.

Mason, L., Stupl, J., Marshall, W., et al. Orbital debris-debris collision avoidance. Adv. Space Res., 48, 1643, 2011.

Norimatsu, T., Kozaki, Y., Miyanaga, N., et al. Conceptual design of laser fusion reactor KOYO-F based on fast ignition scheme. in: Proceedings of the 21st IAEA Conference, (International Atomic Energy Agency, 2008), FT/P5-39, 2008.

Phipps, C.R., Turner, T.P., Harrison, R.F., et al. Impulse coupling to targets in vacuum by $\mathrm{KrF}, \mathrm{HF}$, and $\mathrm{CO} 2$ single-pulse lasers. J. Appl. Phys., 64, 1083-1096, 1988.

Phipps, C.R., Friedman, H., Gavel, D., et al. ORION: Clearing near-Earth space debris using a 20-kW, 530-nm, Earth-based, repetitively pulsed laser. Laser and Particle Beams, 14 (1), 1996.

Phipps, C.R. ORION: Challenges and Benefits. SPIE 3343, Proc. Santa Fe High Power Laser Ablation Conference, 575-582, 1998.

Phipps, C.R., and Sinko, J. Applying new laser interaction models to the ORION problem. AIP Conference Proceedings 1278, 492-501, 2010.

Phipps, C.R., Baker, K.L., Libby, S.B., et al. Removing orbital debris with lasers. Adv. Space Res., 49, 1283, 2012.

Praly, N., Bristeau, P. Petit, N. Study on the eddy current damping of the spin dynamics of space debris. Paper 7.3, 2d European Conference on Active Space Debris Removal, CNES, Paris, 18-19 June 2012.

Rubenchik, A.M., Liedahl, D.A., Erlandson, A.C. Laser system for space debris cleaning. AIP Conf. Proc. 1464, 448-455, 2012.

Schall, W.O. Orbital debris removal by laser radiation. Acta Astronautica, 24, 343, 1991.

Schall, W.O. Removal of small orbital debris with orbiting lasers. SPIE 3343, Proc. Santa Fe High Power Laser Ablation Conference, 564, 1998. 
Sdunnus, H., Bendisch, J., Bunte, K.D., et al. An introduction to the upgraded ESA master model. European Conference on Spacecraft Structures, Materials, and Mechanical Testing, eds., C. Stavrinidis, A. Rolfo, and E. Breitbach, ESASP-468, 2001.

Siegman, A.E. High-power laser beams: Defining, measuring and optimizing transverse beam quality. SPIE 1810 Proc Ninth International Symposium on Gas Flow and Chemical Lasers, 758-765, 1993.

Sinko, J., and Phipps, C. Modeling $\mathrm{CO}_{2}$ laser ablation impulse of polymers in vapor and plasma regimes. Appl. Phys. Lett., 95, 131105, 2009.

Wiedemann, C., Oswald, M., Stabroth, S., et al. Size distribution of NaK droplets released during RORSAT reactor core ejection. Adv. Space Res., 35, 1290, 2005.

Zeldovich, Ya. B., and Raizer, Yu. P. Physics of Shock Waves and HighTemperature Hydrodynamic Phenomena. Dover Publications, Inc. (Mineola, New York), 2002. 